\title{
Material-orientation optimization for tailoring thermal deformation of laminated composite shell structures using a parameter-free approach
}

\author{
Masatoshi SHIMODA*, Yoshiaki MURAMATSU** and Motoki UMEMURA* \\ * Department of Advanced Science and Technology, Toyota Technological Institute \\ 2-12-1 Hisakata, Tenpaku-ku, Nagoya, Aichi 468-8511, Japan \\ E-mail: shimoda@toyota-ti.ac.jp \\ ** Department of Advanced Science and Technology, Graduate School of Engineering, Toyota Technological Institute \\ 2-12-1 Hisakata, Tenpaku-ku, Nagoya, Aichi 468-8511, Japan
}

Received: 22 July 2019; Revised: 14 November 2019; Accepted: 22 November 2019

\begin{abstract}
In this study, we present a material-orientation optimization method for tailoring the thermal displacements of laminated composite shell structures to required target values. The square displacement error norm between the displacements and the target values is minimized by varying the distributed anisotropic material orientation of each layer. The optimum design problem is formulated as a distributed-parameter, or a design parameterization-free optimization problem, and the sensitivity function with respect to the material orientation variation is theoretically derived based on the variational method. The optimal material orientation variation is determined by the $\mathrm{H}^{1}$ gradient method with Poisson's equation, where the sensitivity function is applied as the Robin condition to vary and optimize the material orientation distribution. Then, the optimal material orientation variation is determined as the temperature distribution by the Poisson's equation, which ensures the continuous material distribution. The optimal design examples show that the proposed optimization method can effectively produce the optimum material orientation with a smooth curvilinear distribution for tailoring thermal or static deformation of a laminated composite shell structure.
\end{abstract}

Keywords : $\mathrm{H}^{1}$ gradient method, Laminated composite, Shell structure, Material orientation, Optimization, Deformation tailoring, Thermal deformation

\section{Introduction}

Laminated composite shell structures are used in a variety of industrial products, especially in vehicles for their lightweight design, where the usage of carbon-fiber-reinforced plastics (CFRP) in shell structures has been increasing, since they have higher specific mechanical performances compared to metals. Moreover, "Automated fiber placement (AFP)" has enabled the manufacture of curvilinear-distributed material orientation, which can pulled out their potential and improve the mechanical performances more sufficiently. Most of the proposed optimization methods for the curvilinear-distributed material orientation design consider maximizing the stiffness to an external force as their design objective. In the practical design of laminated shell structures, deformation tailoring, (or controlling the displacements to desired values) to thermal loading has to also be needed to achieve high quality, and to maintain mechanical performances and functions required, since most of the industrial products are exposed to severe temperature environments. Especially, laminated composite shell structures deform unusually due to the coupling and the cross-elasticity effects. It is extremely important for designers to avoid this problem, or tailor the thermal deformation of the laminated composite shell structures.

The deformation tailoring was called "homology design" or "compliant mechanism design" in the previous papers. The concept of homology design was presented for the design of large radio telescopes by Hoerner (1967), where the 
deformation of the structure was defined as homologous if a given geometrical relation holds for the deformation. Later Yoshikawa and Nakagiri (1995) proposed a formulation based on the finite element sensitivity analysis for homology design of frame structures. Shimoda et al. (1998) presented a shape optimization method of continua for homologous deformation using the traction method, and Liu and Shimoda (2014) developed a shape optimization method of a shell structure for achieving the desired deformation mode. Lee et al. (1998) developed a truss optimization method using equality equations to include homology constraints under multiple loading conditions as well as single loading conditions. Another approach for controlling the deformation is based on the optimal design of a compliant mechanism. In designing a compliant mechanism, the displacements of the loading area and the specified region are enforced to reach a desired value in a specified direction, in order to achieve a flexible structure with mechanical functions. Although the objective of the compliant mechanism design is similar to the homology design, the design methods for compliant mechanisms are mainly based on topology optimization. Design of compliant mechanism using topology optimization was first introduced by Ananthasuresh et al. (1994). Subsequently, studies on compliant mechanism with topology optimization were extended to various optimization formulations (Nishiwaki et al. (1998); Jung and Gea (2004); Lee and Gea (2013)). However, studies on the homology design or the compliant mechanism are limited and not reported for laminated shell structures.

Focusing on material orientation optimization, many methods and their application techniques for plates or shells have been reported (e.g. Muramatsu and Shimoda (2019)). The representative optimization methods are a sensitivity based method (Hyer and Lee (1991); (Lindgaard and Lund (2011)), a discrete material optimization method (DMO) (Stegmann and Lund (2009)), a normal distribution fiber optimization method (NDFO) (Kiyono et al. (2017)) and genetic algorithm (GA) based methods (Le and Haftka (1993); (Kogiso et al. (1994); (Kim et al. (1999); (Honda et al. (2013); Guanxin et al. (2016); Jacob and Senthil (2006)). Yamanaka et al. (2016) presented a method using fiber-path parameterization based on stream lines of perfect flow. Albazzan et al. (2019) reviewed on material-orientation optimization using lamination parameters. The sensitivity-based approach, which has mathematical backgrounds, may cause multiple local minima including the unsmooth material distributions, and it is highly dependent on the initial material orientation distribution. On the other hand, DMO and NDFO methods are ameliorations of alternative methods such as simple-gradient methods that may cause multiple local minima, in which the element-based penalization coefficient is used to force the candidate orientations to only one candidate. These methods are categorized into a parametric method. The design variable parameterization is effective for reducing the design freedom, for reducing the risk of obtaining local minima and results in a lower computational cost; however, the obtained mechanical performance is limited since the optimal solution is highly dependent on the pre-defined parameters. GA is also expected to find some local minima and has the potential to provide the global minimum solution, where sensitivity analysis is unnecessary. On the other hand, the application of GA to practical structural problems is limited since FE analyses of large-scale structures with a large number of design variables results in a significant calculation cost, especially for every element in every layer of a laminated shell. In addition, considering a parameter-free orientation design problem, or an enormous design degrees of freedom design problem, it is not easy to control their behaviors. Without a countermeasure, the ill-condition like the checkerboard problem of topology optimization or the jagging problem of shape optimization may happen.

On the contrary, the authors proposed a parameter-free gradient-based material orientation optimization method based on the $\mathrm{H}^{1}$ gradient method for compliance minimization problem of single layer shells (Muramatsu and Shimoda (2017) and of laminated shells (Muramatsu and Shimoda (2019), in which the optimal material distribution can be obtained without design variable parameterization such as the candidate material orientations or the polynomials to express the material distribution. In other words, the materials are varied freely over the entire region with the largest design freedom. The proposed method overcame the issues including the ill-condition risk aforementioned. The main advantages of the $\mathrm{H}^{1}$ gradient methods are that it can conventionally determine the smooth distribution of the design variables and decrease the objective function simultaneously without design variable parameterization.

By developing the prior research (Muramatsu and Shimoda (2019) under these backgrounds, we propose a novel parameter-free material orientation optimization method for tailoring the thermal displacements of laminated composite shell structures with anisotropic materials to the target values in this study. The square displacements error norm between the thermal displacements and the target values is minimized by varying the distributed material orientations of each layer under the state-equation constraint. The arbitrary and optimal material orientation distribution of each 
layer is determined with the $\mathrm{H}^{1}$ gradient method with Poisson's equation we proposed (Muramatsu and Shimoda (2019). This method is applied to several design examples to confirm the effectiveness.

In Section 2, we introduce the governing equation of a laminated shell structure and derive the sensitivity function of the thermal-deformation tailoring problem via the formulation of the thermal-deformation tailoring problem. In Section 3, we introduce the $\mathrm{H}^{1}$ gradient method with Poisson's equation for the free-material orientation problem. In Section 4, we present several numerical design examples and evaluate the results. Finally, the conclusions are summarized in Section 5. In Appendix, we introduce the basic stress-strain relationship of the orthotropic material used in this study.

\section{Formulation of material orientation optimization problem}

\subsection{Governing equation for a laminated shell structure}

As shown in Fig. 1, a laminated shell structure consists of $N$ layers, and the $i$-th ply of a laminated shell structure has an initial design domain $\Omega^{(i)}$, middle surface $A^{(i)}$ with boundary $\partial A^{(i)}$, and thickness $t^{(i)}$. The bounded domain of each layer is composed of a set of infinitesimal flat surfaces $d A^{(i)}$.

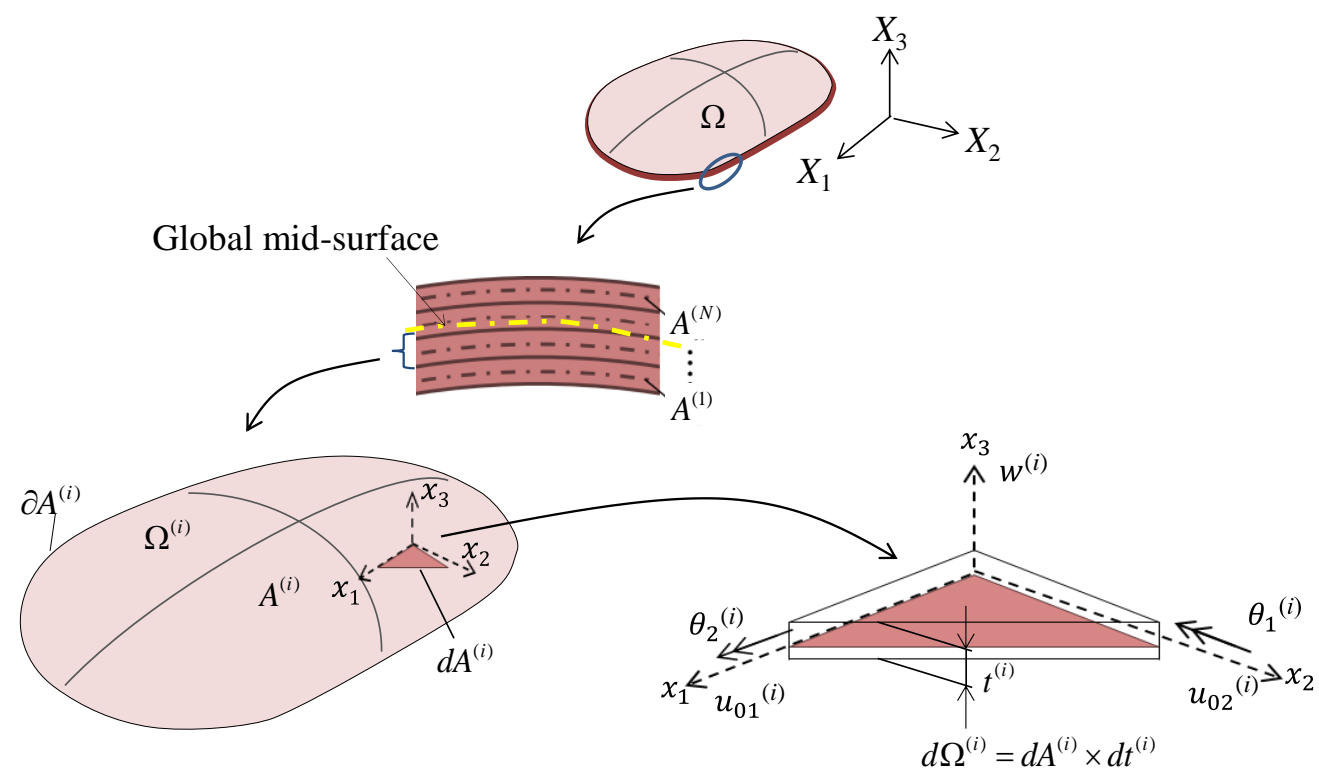

Fig. 1 Laminated shell with $\mathrm{N}$ layers and DOF on local coordinates

It is assumed that the mapping of the local coordinate system $\left(x_{1}, x_{2}, 0\right)$, which gives the position of the global mid-surface of the laminated shell structure, to the global coordinate system $\left(X_{1}, X_{2}, X_{3}\right)$ is piecewise smooth. The Mindlin-Reissner plate theory is employed for plate bending. Using the sign convention on the small triangle domain $d \Omega^{(i)}\left(=d A^{(i)} \times t^{(i)}\right)$ in Fig. 1, the displacements of $i$-th ply expressed by the local coordinates $\boldsymbol{u}^{(i)}=\left\{u_{k}^{(i)}\right\}_{k=1,2,3}$ are considered by dividing them into the displacements in the in-plane direction $\left\{u_{\alpha}^{(i)}\right\}_{\alpha=1,2}$ and the displacements in the out-of-plane direction $u_{3}^{(i)}$, shown as

$$
\begin{aligned}
& u_{\alpha}^{(i)}\left(x_{1}^{(i)}, x_{2}^{(i)}, x_{3}^{(i)}\right) \equiv u_{0 \alpha}^{(i)}\left(x_{1}^{(i)}, x_{2}^{(i)}\right)-x_{3}^{(i)} \theta_{\alpha}^{(i)}\left(x_{1}^{(i)}, x_{2}^{(i)}\right), \\
& u_{3}^{(i)}\left(x_{1}^{(i)}, x_{2}^{(i)}, x_{3}^{(i)}\right) \equiv w^{(i)}\left(x_{1}^{(i)}, x_{2}^{(i)}\right),
\end{aligned}
$$

where $\left\{u_{0 \alpha}^{(i)}\right\}_{\alpha=1,2}, w^{(i)}$ and $\left\{\theta_{\alpha}^{(i)}\right\}_{\alpha=1,2}$ express in-plane displacement, out-of-plane displacement and rotational angles of the mid-area of the $i$-th ply of the laminated shell structure, respectively. Then, the weak form state equation with respect to $\boldsymbol{u}^{(i)}=\left(\boldsymbol{u}_{0}^{(i)}, w^{(i)}, \boldsymbol{\theta}^{(i)}\right),(i=1, \cdots, N)$ can be expressed as Eq. (3) by substituting Eq. (1) and (2) into the variational equation of the three-dimensional linear elastic body, considering $\sigma_{33}^{(i)}=0$ and eliminating $\varepsilon_{33}^{(i)}$. 


$$
\begin{array}{r}
a\left(\left(\boldsymbol{u}^{(1)}, \boldsymbol{u}^{(2)}, \cdots, \boldsymbol{u}^{(N)}\right),\left(\overline{\boldsymbol{u}}^{(1)}, \overline{\boldsymbol{u}}^{(2)}, \cdots, \overline{\boldsymbol{u}}^{(N)}\right)\right)=l\left(\overline{\boldsymbol{u}}^{(1)}, \overline{\boldsymbol{u}}^{(2)}, \cdots, \overline{\boldsymbol{u}}^{(N)}\right), \quad \forall\left(\overline{\boldsymbol{u}}^{(1)}, \overline{\boldsymbol{u}}^{(2)}, \cdots, \overline{\boldsymbol{u}}^{(N)}\right) \in \bar{U}, \\
\boldsymbol{u}^{(i)}=\left(\boldsymbol{u}_{0}^{(i)}, w^{(i)}, \boldsymbol{\theta}^{(i)}\right) \in U \quad(i=1, \cdots, N),
\end{array}
$$

where $\boldsymbol{u}^{(i)}=\left[\boldsymbol{u}_{0}^{(i)}, w^{(i)}, \boldsymbol{\theta}^{(i)}\right]^{T}$, and $(\cdot)$ expresses a variation. In addition, the bilinear form $a(\cdot, \cdot)$ and linear form $l(\cdot)$ for the state variables $\left(\boldsymbol{u}_{0}, w, \boldsymbol{\theta}\right)$ are respectively defined as

$$
\begin{aligned}
& a\left(\left(\boldsymbol{u}^{(1)}, \boldsymbol{u}^{(2)}, \cdots, \boldsymbol{u}^{(N)}\right),\left(\overline{\boldsymbol{u}}^{(1)}, \overline{\boldsymbol{u}}^{(2)}, \cdots, \overline{\boldsymbol{u}}^{(N)}\right)\right)=\sum_{i=1}^{N} \int_{A_{i}}\left\{\left\{\boldsymbol{\kappa}^{(i) T}(\boldsymbol{\theta})-\boldsymbol{\alpha}^{T} \frac{T_{g}^{(i)}}{t_{i}}\right\} \boldsymbol{E}^{B(i)} \overline{\boldsymbol{\kappa}}^{(i)}(\overline{\boldsymbol{\theta}})\right. \\
& +\left\{\boldsymbol{\varepsilon}_{0}^{(i) T}\left(\boldsymbol{u}_{0}\right)-\boldsymbol{\alpha}^{T} T_{m}^{(i)}\right\}\left(\boldsymbol{u}_{0}\right) \boldsymbol{E}^{M(i)} \overline{\boldsymbol{\varepsilon}}_{0}^{(i)}\left(\overline{\boldsymbol{u}}_{0}\right)+\left\{\boldsymbol{\kappa}^{(i) T}(\boldsymbol{\theta})-\boldsymbol{\alpha}^{T} \frac{T_{g}^{(i)}}{t_{i}}\right\} \boldsymbol{E}^{C(i)} \overline{\boldsymbol{\varepsilon}}_{0}^{(i)}\left(\overline{\boldsymbol{u}}_{0}\right) \\
& +\overline{\boldsymbol{\kappa}}^{(i) T}(\overline{\boldsymbol{\theta}}) \boldsymbol{E}^{C(i)}\left\{\boldsymbol{\varepsilon}_{0}^{(i)}\left(\boldsymbol{u}_{0}\right)-\boldsymbol{\alpha} T_{m}^{(i)}\right\}+\left\{\boldsymbol{\varepsilon}_{0}^{(i) T}\left(\boldsymbol{u}_{0}\right)-\boldsymbol{\alpha}^{T} T_{m}^{(i)}\right\} \boldsymbol{E}^{C(i)} \overline{\boldsymbol{\kappa}}^{(i)}(\overline{\boldsymbol{\theta}}) \\
& \left.+\overline{\boldsymbol{\varepsilon}}_{0}^{(i) T}\left(\overline{\boldsymbol{u}}_{0}\right) \boldsymbol{E}^{C(i)}\left\{\boldsymbol{\kappa}^{(i)}(\boldsymbol{\theta})-\boldsymbol{\alpha} \frac{T_{g}^{(i)}}{t_{i}}\right\}+\boldsymbol{\gamma}^{(i) T}(w, \boldsymbol{\theta}) \boldsymbol{E}^{S(i)} \bar{\gamma}^{(i)}(\bar{w}, \overline{\boldsymbol{\theta}})\right\} d A, \\
& l\left(\overline{\boldsymbol{u}}^{(1)}, \overline{\boldsymbol{u}}^{(2)}, \cdots, \overline{\boldsymbol{u}}^{(N)}\right)=\sum_{i=1}^{N}\left[\int_{A_{i}}\left(\boldsymbol{f}^{T} \overline{\boldsymbol{u}}_{0}^{(i)}-\boldsymbol{m}^{T} \overline{\boldsymbol{\theta}}^{(i)}+q \bar{w}^{(i)}\right) d A+\int_{\partial A_{i}}\left(\boldsymbol{N}^{T} \overline{\boldsymbol{u}}_{0}^{(i)}-\boldsymbol{M}^{T} \overline{\boldsymbol{\theta}}^{(i)}+Q \bar{w}^{(i)}\right) d s\right] .
\end{aligned}
$$

The external loadings relative to the local coordinate system $\left(x_{1}, x_{2}, 0\right)$ are defined as $\boldsymbol{f}, \boldsymbol{m}, q, \boldsymbol{N}, \boldsymbol{M}$ and $Q$, which denote in-plane load, out-of-plane moment, out-of-plane load on the global middle surface, in-plane load, bending moment, and shearing force on the global sub-boundaries, respectively. The notations $\boldsymbol{E}^{B(i)}, \boldsymbol{E}^{M(i)}, \boldsymbol{E}^{C(i)}$ and $\boldsymbol{E}^{S(i)}$ express the orthotropic elastic matrices with respect to bending, membrane, coupling and shear component of the $i$-th ply, respectively. $\alpha=\left\{\delta_{i j} \alpha_{i j}\right\}_{i, j=1,2}, T_{m}^{(i)}$ and $T_{g}^{(i)} / t^{(i)}$ indicate coefficients of thermal expansion, mean temperature difference and temperature gradient, respectively. Additionally, $\boldsymbol{\varepsilon}^{(i)}=\left\{\varepsilon_{\alpha \beta}\right\}_{\alpha, \beta=1,2}^{(i)}, \quad \boldsymbol{\kappa}^{(i)}=\left\{\kappa_{\alpha \beta}\right\}_{\alpha, \beta=1,2}^{(i)}$, $\boldsymbol{\varepsilon}_{0}^{(i)}=\left\{\varepsilon_{0 \alpha \beta}\right\}_{\alpha, \beta=1,2}^{(i)}$ and $\gamma^{(i)}=\left\{\gamma_{\alpha 3}\right\}_{\alpha=1,2}^{(i)}$ express strain tensor, curvature tensor, in-plane strain tensor and transverse shear strain vector on the middle surface of the $i$-th ply, respectively; and they are defined by the following equations:

$$
\begin{aligned}
\varepsilon_{\alpha \beta}^{(i)} & \equiv \frac{1}{2}\left(u_{\alpha, \beta}^{(i)}+u_{\beta, \alpha}^{(i)}\right), \\
u_{\alpha, \beta}^{(i)} & =u_{0 \alpha, \beta}^{(i)}-x_{3}^{(i)} \theta_{\alpha, \beta}^{(i)}, \\
\gamma_{\alpha}^{(i)} & \equiv w_{, \alpha}^{(i)}-\theta_{\alpha}^{(i)}, \\
\kappa_{\alpha \beta}^{(i)} & \equiv \frac{1}{2}\left(\theta_{\alpha, \beta}^{(i)}+\theta_{\beta, \alpha}^{(i)}\right), \\
\varepsilon_{0 \alpha \beta}^{(i)} & \equiv \frac{1}{2}\left(u_{0 \alpha, \beta}^{(i)}+u_{0 \beta, \alpha}^{(i)}\right) .
\end{aligned}
$$

The displacement continuity and the Cauchy stress vector between each layer are satisfied as

$$
\begin{aligned}
& \boldsymbol{u}^{(i) \text { bottom }}=\boldsymbol{u}^{(i-1) \text { top }}, \\
& \boldsymbol{n}^{(i) \text { bottom }}=-\boldsymbol{n}^{(i-1) \text { top }}, \sigma^{T(i) b o t t o m} \boldsymbol{n}^{(i) b o t t o m}=-\boldsymbol{\sigma}^{T(i-1) \text { top }} \boldsymbol{n}^{(i-1) \text { top },}
\end{aligned}
$$

where $(\cdot)^{(i) b o t t o m}$ and $(\cdot)^{(i-1) \text { top }}$ indicate the value on the bottom surface of the $i$-th ply and the top surface of the (i-1)-th ply, respectively. In addition, $\boldsymbol{n}$ and $\boldsymbol{\sigma}$ are the unit outward normal vector and the Cauchy stress tensor, respectively. Note that, in this paper, the subscripts of the Greek letters are expressed as $\alpha=1,2$ and the tensor subscript notation uses Einstein's summation convention and a partial differential notation with respect to the spatial coordinates $(\cdot)_{, j}=\partial(\cdot) / \partial x_{j}$. 
It will be also noted that $U$ and $\bar{U}$ in Eq. (3) are the spaces of the kinematically admissible displacements given by the following equations:

$$
\begin{array}{r}
U=\left\{\left(u_{01}^{(i)}, u_{02}^{(i)}, w^{(i)}, \theta_{1}^{(i)}, \theta_{2}^{(i)}\right) \in\left(H^{1}(A)\right)^{5} \mid\right. \text { satisfying the constraint conditon appropriately on the } \\
\text { Dirichlet boundary }\}, \\
\bar{U}=\left\{\left(\bar{u}_{01}^{(i)} \bar{u}_{02}^{(i)}, \bar{w}^{(i)}, \bar{\theta}_{1}^{(i)}, \bar{\theta}_{2}^{(i)}\right) \in\left(H^{1}\left(A_{i}\right)\right)^{5},(i=1, \cdots, N) \mid \begin{array}{l}
\bar{u}_{01}^{(i)}=\bar{u}_{02}^{(i)}=\bar{w}^{(i)}=\bar{\theta}_{1}^{(i)}=\bar{\theta}_{2}^{(i)}=0 \\
\text { on each Dirichlet subboundary }\},
\end{array}\right.
\end{array}
$$

where $H^{1}\left(=W^{1,2}\right)$ is the Sobolev space of square integrable and differentiable of order 1.

\subsection{Formulation of tailoring problem of thermal or static deformation}

We aim at tailoring the thermal or static displacements of a laminated shell structure to desired target values by optimizing the material orientations of every layer. Using the state equation as the constraint condition, and square displacement error norm between the displacements and the target values as the objective function to be minimized, a distributed-parameter optimization problem for determining the optimal distribution of the material orientation is formulated as

$$
\begin{array}{ll}
\text { Find } & \delta \varphi^{(i)}(\boldsymbol{x}), \\
\text { that minimizes } & d\left(\boldsymbol{u}^{(M)}-\hat{\boldsymbol{u}}^{(M)}, \boldsymbol{u}^{(M)}-\hat{\boldsymbol{u}}^{(M)}\right), \\
\text { subject to } & \text { Eq. (3), }
\end{array}
$$

where $\delta \varphi^{(i)}(\boldsymbol{x}),\left(\boldsymbol{x} \in A^{(i)}\right)$ is the variation of the material orientation from its original material orientation function $\varphi^{(i)}(\boldsymbol{x})$ of $E_{1}^{(i)}$ on the $i$-th ply, and the updated material orientation function $\varphi_{S}^{(i)}(\boldsymbol{x})$ is described as $\varphi_{s}^{(i)}(\boldsymbol{x})=\varphi^{(i)}(\boldsymbol{x})+\delta \varphi^{(i)}(\boldsymbol{x}) \quad$ (shown in Fig. 2). $\boldsymbol{u}^{(M)}$ and $\hat{\boldsymbol{u}}^{(M)}$ indicate the actual displacement and the target displacement of the control points at the global mid-surface of laminated shell structure, respectively. Eq. (16) shows the square displacement error norm between $\boldsymbol{u}^{(M)}$ and $\hat{\boldsymbol{u}}^{(M)}$.

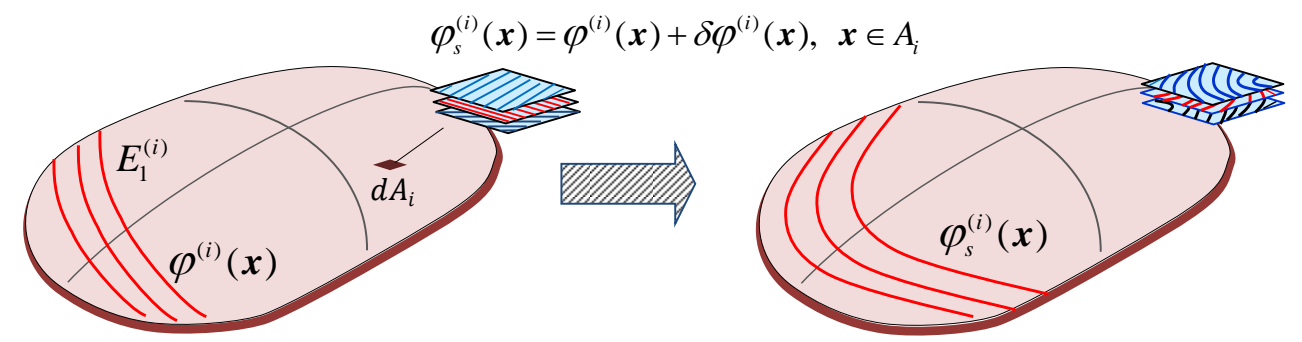

Fig. 2 Variation of material orientation of orthotropic material of the $i$-th ply

\subsection{Derivation of sensitivity function with respect to variation of material orientation function}

The Lagrange multiplier method is used to transform this constrained material orientation optimization problem to a non-constrained material orientation optimization problem. Letting $\left(\overline{\boldsymbol{u}}_{0}^{(i)}, \overline{\boldsymbol{w}}^{(i)}, \overline{\boldsymbol{\theta}}^{(i)}\right)$ denote the Lagrange multiplier with respect to the strong formed state equation, the Lagrange functional $L$ associated with this problem is expressed as

$$
\begin{array}{r}
L\left(\left(\boldsymbol{u}^{(1)}, \boldsymbol{u}^{(2)}, \cdots, \boldsymbol{u}^{(N)}\right),\left(\overline{\boldsymbol{u}}^{(1)}, \overline{\boldsymbol{u}}^{(2)}, \cdots, \overline{\boldsymbol{u}}^{(N)}\right)\right)=d\left(\boldsymbol{u}^{(M)}-\hat{\boldsymbol{u}}^{(M)}, \boldsymbol{u}^{(M)}-\hat{\boldsymbol{u}}^{(M)}\right)+l\left(\overline{\boldsymbol{u}}^{(1)}, \overline{\boldsymbol{u}}^{(2)}, \cdots, \overline{\boldsymbol{u}}^{(N)}\right) \\
-a\left(\left(\boldsymbol{u}^{(1)}, \boldsymbol{u}^{(2)}, \cdots, \boldsymbol{u}^{(N)}\right),\left(\overline{\boldsymbol{u}}^{(1)}, \overline{\boldsymbol{u}}^{(2)}, \cdots, \overline{\boldsymbol{u}}^{(N)}\right)\right) .
\end{array}
$$

Using the variation of $\varphi^{(i)}(\boldsymbol{x})$, i.e. the design variable field $\delta \varphi^{(i)}(\boldsymbol{x})$ to represent the amount of the material orientation variation, the first variation $\delta L$ of the Lagrange functional $L$ with respect to the material orientation variation can be expressed as 


$$
\begin{aligned}
& \delta L=l\left(\overline{\boldsymbol{u}}^{\prime(1)}, \overline{\boldsymbol{u}}^{(2)}, \cdots, \overline{\boldsymbol{u}}^{(N)}\right)-a\left(\left(\boldsymbol{u}^{\prime(1)}, \boldsymbol{u}^{\prime(2)}, \cdots, \boldsymbol{u}^{(N)}\right),\left(\overline{\boldsymbol{u}}^{(1)}, \overline{\boldsymbol{u}}^{(2)}, \cdots, \overline{\boldsymbol{u}}^{(N)}\right)\right) \\
& \quad-a\left(\left(\boldsymbol{u}^{(1)}, \boldsymbol{u}^{(2)}, \cdots, \boldsymbol{u}^{(N)}\right),\left(\overline{\boldsymbol{u}}^{\prime(1)}, \overline{\boldsymbol{u}}^{\prime(2)}, \cdots, \overline{\boldsymbol{u}}^{\prime(N)}\right)\right)+2 d\left(\boldsymbol{u}^{(M)}-\hat{\boldsymbol{u}}^{(M)}, \boldsymbol{u}^{(M)^{\prime}}\right)+\left\langle G_{\varphi}^{(i)}, \delta \varphi^{(i)}\right\rangle_{\varphi},
\end{aligned}
$$

where

$$
\begin{aligned}
&\left\langle G_{\varphi}^{(i)}, \delta \varphi^{(i)}\right\rangle_{\varphi}=\sum_{i=1}^{N} \int_{A_{i}} G_{\varphi}^{(i)} \delta \varphi^{(i)} d A \\
&=\sum_{i=1}^{N} \int_{A_{i}}\left[\left\{\boldsymbol{\kappa}^{(i) T}(\boldsymbol{\theta})-\boldsymbol{\alpha}^{T} \frac{T_{g}^{(i)}}{t^{(i)}}\right\} \frac{\partial \boldsymbol{E}^{B(i)}}{\partial \varphi} \overline{\boldsymbol{\kappa}}^{(i)}(\overline{\boldsymbol{\theta}})+\left\{\boldsymbol{\varepsilon}_{0}^{(i) T}\left(\boldsymbol{u}_{0}\right)-\boldsymbol{\alpha}^{T} T_{m}^{(i)}\right\} \frac{\partial \boldsymbol{E}^{M(i)}}{\partial \varphi} \overline{\boldsymbol{\varepsilon}}_{0}^{(i)}\left(\overline{\boldsymbol{u}}_{0}\right)\right. \\
&+\left\{\boldsymbol{\kappa}^{(i) T}(\boldsymbol{\theta})-\boldsymbol{\alpha}^{T} \frac{T_{g}^{(i)}}{\left.t^{(i)}\right\}}\right\} \frac{\partial \boldsymbol{E}^{\mathrm{C}(i)}}{\partial \varphi} \overline{\boldsymbol{\varepsilon}}_{0}^{(i)}\left(\overline{\boldsymbol{u}}_{0}\right)+\overline{\boldsymbol{\kappa}}^{(i) T}(\overline{\boldsymbol{\theta}}) \frac{\partial \boldsymbol{E}^{\mathrm{C}(i)}}{\partial \varphi}\left\{\boldsymbol{\varepsilon}_{0}^{(i)}\left(\boldsymbol{u}_{0}\right)-\boldsymbol{\alpha} T_{m}^{(i)}\right\} \\
&+\left\{\boldsymbol{\varepsilon}_{0}^{(i) T}\left(\boldsymbol{u}_{0}\right)-\boldsymbol{\alpha}^{T} T_{m}^{(i)}\right\} \frac{\partial \boldsymbol{E}^{\mathrm{C}(i)}}{\partial \varphi} \overline{\boldsymbol{\kappa}}^{(i)}(\overline{\boldsymbol{\theta}})+\overline{\boldsymbol{\varepsilon}}_{0}^{(i) T}\left(\overline{\boldsymbol{u}}_{0}\right) \frac{\partial \boldsymbol{E}^{\mathrm{C}(i)}}{\partial \varphi}\left\{\boldsymbol{\kappa}^{(i)}(\boldsymbol{\theta})-\boldsymbol{\alpha} \frac{T_{g}^{(i)}}{\left.t^{(i)}\right\}}\right. \\
&\left.+\boldsymbol{\gamma}^{(i) T}(w, \boldsymbol{\theta}) \frac{\partial \boldsymbol{E}^{\mathrm{S}(i)}}{\partial \varphi} \bar{\gamma}^{(i)}(\bar{w}, \overline{\boldsymbol{\theta}})\right] \delta \varphi^{(i)} d A,
\end{aligned}
$$

and where $(\cdot)^{\prime}$ indicates the first variation with respect to the design variable field $\delta \varphi^{(i)}(\boldsymbol{x})$.

When Eqs. (21) and (22) are satisfied,

$$
\begin{aligned}
& a\left(\left(\boldsymbol{u}^{(1)}, \boldsymbol{u}^{(2)}, \cdots, \boldsymbol{u}^{(N)}\right),\left(\overline{\boldsymbol{u}}^{\prime(1)}, \overline{\boldsymbol{u}}^{(2)}, \cdots, \overline{\boldsymbol{u}}^{(N)}\right)\right)=l\left(\overline{\boldsymbol{u}}^{\prime(1)}, \overline{\boldsymbol{u}}^{\prime(2)}, \cdots, \overline{\boldsymbol{u}}^{(N)}\right), \\
& \left(\boldsymbol{u}^{(1)}, \boldsymbol{u}^{(2)}, \cdots, \boldsymbol{u}^{(N)}\right) \in U, \forall\left(\overline{\boldsymbol{u}}^{(1)}, \overline{\boldsymbol{u}}^{(2)}, \cdots, \overline{\boldsymbol{u}}^{(N)}\right) \in \bar{U}, \\
& a\left(\left(\boldsymbol{u}^{\prime(1)}, \boldsymbol{u}^{\prime(2)}, \cdots, \boldsymbol{u}^{\prime(N)}\right),\left(\overline{\boldsymbol{u}}^{(1)}, \overline{\boldsymbol{u}}^{(2)}, \cdots, \overline{\boldsymbol{u}}^{(N)}\right)\right)=2 d\left(\boldsymbol{u}^{(M)}-\hat{\boldsymbol{u}}^{(M)}, \boldsymbol{u}^{(M) \prime}\right), \\
& \left(\overline{\boldsymbol{u}}^{(1)}, \overline{\boldsymbol{u}}^{(2)}, \cdots, \overline{\boldsymbol{u}}^{(N)}\right) \in \bar{U}, \forall\left(\boldsymbol{u}^{(1)}, \boldsymbol{u}^{\prime(2)}, \cdots, \boldsymbol{u}^{\prime(N)}\right) \in U,
\end{aligned}
$$

then Eq. (19) becomes

$$
\delta L=\left\langle G_{\varphi}^{(i)}, \delta \varphi^{(i)}\right\rangle_{\varphi}
$$

Equation (21) is the governing equation for the state variable $\boldsymbol{u}^{(i)}=\left(\boldsymbol{u}_{0}^{(i)}, w^{(i)}, \boldsymbol{\theta}^{(i)}\right),(i=1,2,3, \cdots, N)$ and coincides with the state equation (Eq. (3)). Eq. (22) is the adjoint equation for the adjoint variable $\overline{\boldsymbol{u}}^{(i)}=\left(\overline{\boldsymbol{u}}_{0}^{(i)}, \overline{\boldsymbol{w}}^{(i)}, \overline{\boldsymbol{\theta}}^{(i)}\right)$, $(i=1,2,3, \cdots, N)$.

Finally, the material orientation sensitivity function $G_{\varphi}^{(i)}$ of this problem is derived as

$$
\begin{aligned}
G_{\varphi}^{(i)}= & \left\{\boldsymbol{\kappa}^{(i) T}(\boldsymbol{\theta})-\boldsymbol{\alpha}^{T} \frac{T_{g}^{(i)}}{t^{(i)}}\right\} \frac{\partial \boldsymbol{E}^{B(i)}}{\partial \varphi} \overline{\boldsymbol{\kappa}}^{(i)}(\overline{\boldsymbol{\theta}})+\left\{\boldsymbol{\varepsilon}_{0}^{(i) T}\left(\boldsymbol{u}_{0}\right)-\boldsymbol{\alpha}^{T} T_{m}^{(i)}\right\} \frac{\partial \boldsymbol{E}^{M(i)}}{\partial \varphi} \overline{\boldsymbol{\varepsilon}}_{0}^{(i)}\left(\overline{\boldsymbol{u}}_{0}\right)+\left\{\boldsymbol{\kappa}^{(i) T}(\boldsymbol{\theta})-\boldsymbol{\alpha}^{T} \frac{T_{g}^{(i)}}{t^{(i)}}\right\} \frac{\partial \boldsymbol{E}^{\mathrm{C}(i)}}{\partial \varphi} \overline{\boldsymbol{\varepsilon}}_{0}^{(i)}\left(\overline{\boldsymbol{u}}_{0}\right) \\
& +\overline{\boldsymbol{\kappa}}^{(i) T}(\overline{\boldsymbol{\theta}}) \frac{\partial \boldsymbol{E}^{\mathrm{C}(i)}}{\partial \varphi}\left\{\boldsymbol{\varepsilon}_{0}^{(i)}\left(\boldsymbol{u}_{0}\right)-\boldsymbol{\alpha} T_{m}^{(i)}\right\}+\left\{\boldsymbol{\varepsilon}_{0}^{(i) T}\left(\boldsymbol{u}_{0}\right)-\boldsymbol{\alpha}^{T} T_{m}^{(i)}\right\} \frac{\partial \boldsymbol{E}^{\mathrm{C}(i)}}{\partial \varphi} \overline{\boldsymbol{\kappa}}^{(i)}(\overline{\boldsymbol{\theta}})+\overline{\boldsymbol{\varepsilon}}_{0}^{(i) T}\left(\overline{\boldsymbol{u}}_{0}\right) \frac{\partial \boldsymbol{E}^{\mathrm{C}(i)}}{\partial \varphi}\left\{\boldsymbol{\kappa}^{(i)}(\boldsymbol{\theta})-\boldsymbol{\alpha} \frac{T_{g}^{(i)}}{t^{(i)}}\right\} \\
& +\boldsymbol{\gamma}^{(i) T}(w, \boldsymbol{\theta}) \frac{\partial \boldsymbol{E}^{\mathrm{S}(i)}}{\partial \varphi} \bar{\gamma}^{(i)}(\bar{w}, \overline{\boldsymbol{\theta}}) .
\end{aligned}
$$

The each term in Eq. (24) is calculated by Eqs. (21) and (22), and the finite element method can be employed to solve those equations. The components of the elastic matrices (Gürdal et al. (1999)) on the right side of Eq. (24) will be introduced in Appendix A1. 
Note that the material orientation sensitivity function for static deformation can be obtained by neglecting the thermal expansion terms of Eq. (24). The numerical example of tailoring static deformation will be presented in Section 4.2.3 along with the numerical examples of tailoring thermal deformation.

When solving the state equation and the adjoint equation using the finite element method, two modeling are conceivable. The first is to use only one layer as an element and find the stress and strain distribution of each layer by using the classical laminate theory. The other uses the multiple elements layer by layer, and the nodes of the elements of each layer are shared, or the multiple layers overlap to model a laminated shell structure. Since the center plane of each layer is offset from the middle plane of the whole plate, it is necessary to consider this offset for the second moment of area of the section given to each layer. In addition, the calculation of stress and strain of each layer also needs to consider the offset. It has been confirmed in a previous study that methods 1 and 2 can produce almost the same calculation results. In this study, we decided to adopt the former method of modeling using classical laminate theory. The derived material orientation sensitivity function $G_{\varphi}^{(i)}$ will be applied to the $\mathrm{H}^{1}$ gradient method with Poisson's equation in Section 3.

\section{3. $\mathbf{H}^{1}$ gradient method with Poisson's equation for free-material orientation optimization}

The original $\mathrm{H}^{1}$ gradient method called traction method at first was proposed for shape optimization of a linear elastic structure by Azegami and $\mathrm{Wu}$ (1994), and Shimoda and Liu (2014) extended it for free-form optimization of shells, which is a gradient method in the Hilbert space, and is theoretically possible to treat infinite design degrees of freedom. In these methods, the optimal distribution of the vector design variable can be determined via a linear elastic equation. In addition, the $\mathrm{H}^{1}$ gradient method is further extended to size optimization (Ikeya et al. (2016)) and topology optimization with SIMP method (Azegami et al. (2011)). The extended $\mathrm{H}^{1}$ gradient methods can determine the optimal distribution of the scalar design variable via Poisson's equation. The authors extended this method moreover for the material-orientation optimization problem (Muramatsu and Shimoda (2019)). The $\mathrm{H}^{1}$ gradient method with Poisson's equation proposed for the optimal material-distribution is as follows:

When the state and the adjoint equations are satisfied, the perturbation expansion $\Delta L$ of Eq. (23) can be expressed as

$$
\Delta L=\left\langle G_{\varphi}^{(i)}, \Delta s \delta \varphi^{(i)}\right\rangle
$$

where $\Delta s$ is a sufficient small positive value.

In order to obtain the optimal material orientation variation field $\delta \varphi^{(i)}(\boldsymbol{x})$ of the $i$-th ply, the following weak-form Poisson's equation for $\delta \varphi^{(i)}(\boldsymbol{x})$ is introduced.

$$
\begin{aligned}
& b\left(\delta \varphi^{(1)}, p^{(1)}, \cdots, \delta \varphi^{(N)}, p^{(N)}\right)+\beta_{\varphi}\left\langle\delta \varphi^{(i)}, v^{(i)}\right\rangle=-\left\langle G_{\varphi}^{(i)}, p^{(i)}\right\rangle, \forall p^{(i)} \in C_{v}, \delta \varphi^{(i)} \in C_{\varphi}, \\
& b\left(\delta \varphi^{(1)}, p^{(1)}, \cdots, \delta \varphi^{(N)}, p^{(N)}\right)=\sum_{i=1}^{N} \int_{A_{i}} \delta \varphi_{, i}^{(i)} k_{i j} p_{, j}^{(i)} d A,
\end{aligned}
$$

where $\delta \varphi^{(i)}(\boldsymbol{x})$ denotes the material orientation field to be determined. The notations $\beta_{\varphi}(>0)$ and $k_{i j}$ are equivalent to the heat transfer coefficient and the thermal conductivity matrix in the heat transfer analysis, respectively. $C_{\varphi}$ is the function space of the kinematically admissible temperatures that satisfy the Dirichlet conditions for material orientation variation $\delta \varphi^{(i)}(\boldsymbol{x})$, and $C_{v}$ is the function space of the kinematically admissible temperatures defined as:

$$
C_{v}=\left\{p^{(i)} \in H^{1} \mid p^{(i)}=0 \text { on the Dirichlet boundaries }\right\} .
$$

The Dirichlet conditions can be arbitrarily defined by only considering the design requirement for the material orientations.

Substituting Eq. (26) into Eq. (25) and considering the arbitrariness of $p^{(i)}$ in Eq. (26), we obtain

$$
\Delta L=\left\langle G_{\varphi}^{(i)}, \Delta s \delta \varphi^{(i)}\right\rangle=-\Delta s\left(b\left(\delta \varphi^{(1)}, \delta \varphi^{(1)}, \cdots, \delta \varphi^{(N)}, \delta \varphi^{(N)}\right)+\beta_{\varphi}\left\langle\delta \varphi^{(i)}, \delta \varphi^{(i)}\right\rangle\right) .
$$


Furthermore, considering the positive definitiveness of $\beta_{\varphi}\left\langle\delta \varphi^{(i)}, \delta \varphi^{(i)}\right\rangle>0$ and $b\left(\delta \varphi^{(1)}, \delta \varphi^{(1)}, \cdots, \delta \varphi^{(N)}, \delta \varphi^{(N)}\right)>0$ in Eq. (28), we have $\Delta L<0$. This relationship holds true in a piecewise convex design space.

As above-mentioned, the sensitivity function is not applied directly to update the material orientation variation but it is once replaced by a fictitious internal heat generation and applied to the design domain. Then, the material orientation variation is determined as the temperature field obtained by the Poisson's equation. This makes it possible both to reduce the objective functional and to maintain the smoothness of the material orientation distribution, simultaneously. $\beta_{\varphi}$ has a role of smoothing filter for controlling the influence range of the sensitivity function at a point. With larger $\beta_{\varphi}$, the influence area of the material orientation sensitivity function is smaller. With smaller $\beta_{\varphi}$, the influence area of the material orientation sensitivity function is larger, and then the material orientation distribution becomes smoother, or the curvature change of the material orientation flow becomes smaller. Generally, there is a trade-off relationship between them. This value is empirically defined based on the numerical experiment in the previous study (Muramatsu and Shimoda (2019)).

Figure 3 shows the schematic flowchart with the concept illustrations of the optimization system developed in this study. The material orientation optimization process is summarized as: (1) Stiffness and adjoint analyses by Eqs. (21) and (22), and evaluation of objective function; (2) Calculation of material orientation sensitivity function by Eq. (24) using Eqs. (21) and (22); (3) The negative material orientation sensitivity function $-G_{\varphi}^{(i)}$ is applied layer by layer as a distributed internal heat generation to a fictitious elastic shell structure to the design surface of the $i$-th ply. The material orientation variation field $\delta \varphi(\boldsymbol{x})$ is obtained as the temperature field solved by the Poisson's equation; (4) Updating of the material orientation by $\delta \varphi(\boldsymbol{x})$. This process is repeated until convergence or a given iteration number. A commercial FEM code is used for the processes in yellow. The optimization system can be easily constructed in combination with a commercial FEA code because the proposed method does not need to manipulate the stiffness matrix in each process.

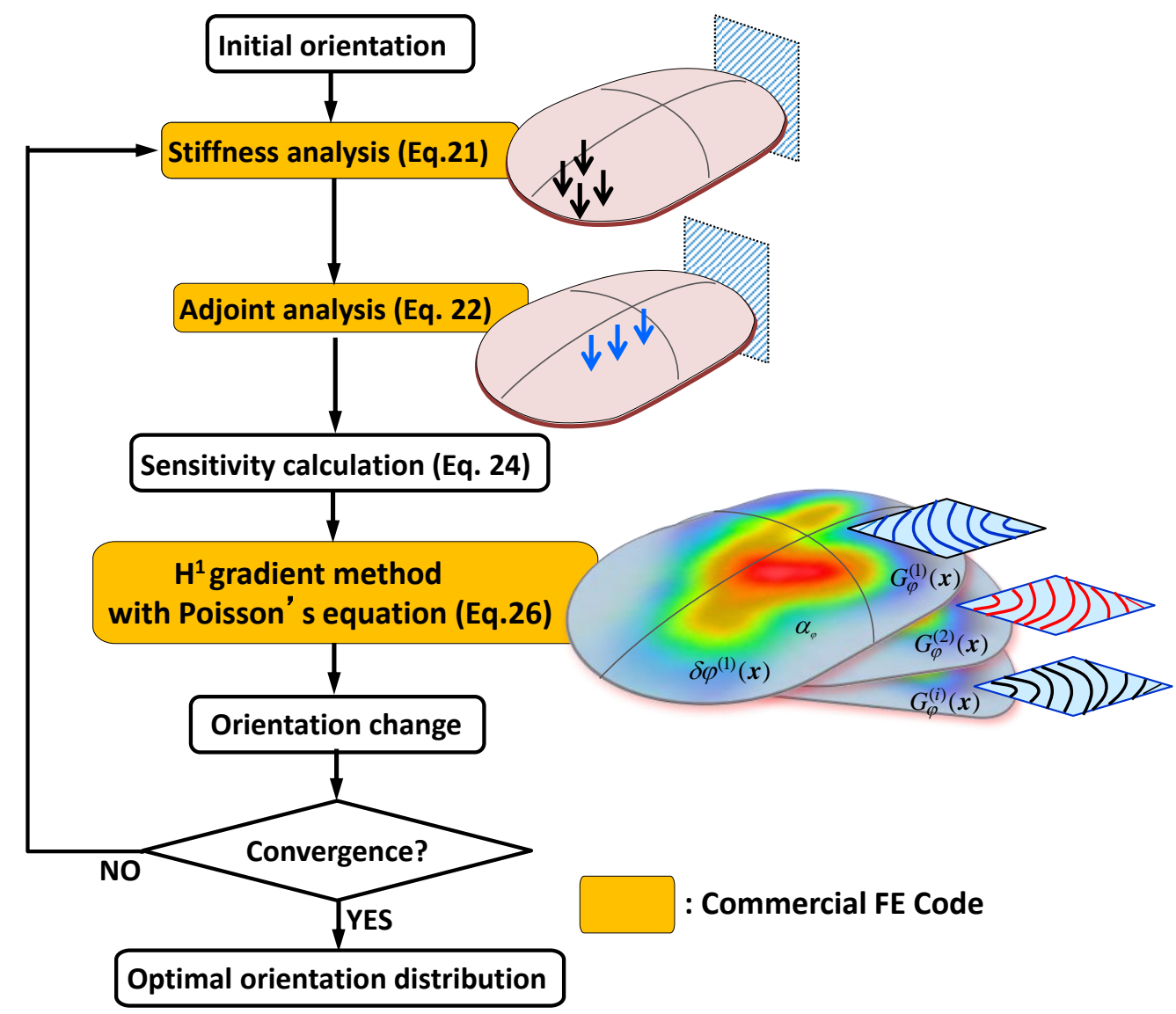

Fig. 3 Schematic flowchart with concept illustration of optimization system 


\section{Numerical examples}

Numerical design examples of a laminated rectangular plate, a laminated square shell, and more practical numerical example of a car hood are shown to evaluate the effectiveness of the proposed method for laminated anisotropic shell structures. Since all the following numerical examples are thin plate problems, the optimization calculations are performed by ignoring the terms on the transverse shear. In all examples, Young's moduli of the orthotropic material $E_{1}$ and $E_{2}$ are respectively assumed to be $210 \mathrm{GPa}$ and $21 \mathrm{GPa}$ virtually. Transverse elasticity modulus is $G_{12}=65 \mathrm{GPa}$ and Poisson ratio is $v_{12}=0.3$. The thickness of each layer is set as $1 \mathrm{~mm}$, and the ratio of each of coefficients of thermal expansion $\alpha_{1}$ and $\alpha_{2}$ is set as $1: 7$. The values of $\beta_{\varphi} \mathrm{W} /\left(\mathrm{mm}^{2} \mathrm{~K}\right)$ and $k_{i j}$ $\mathrm{W} /(\mathrm{mmK})$ in Eq.(26) are set as 0.5 and 2.0, respectively.

\subsection{Three-layer rectangular plate}

It is known that uniform thermal loading to unsymmetrically laminated plates causes unintentional or harmful curvature deformation by the bending-membrane coupling or the cross-elasticity effect. We apply the proposed method to this behavior in order to verify the effectiveness of the proposed method. Figure 4 (a) shows an unsymmetrically laminated plate with 3 layers, which consists of 3 layers with 3 different orientations $\left[45^{\circ} / 90^{\circ} / 0^{\circ}\right]$. The design domain is rectangular of size $100 \times 200 \mathrm{~mm}$, which has 800 triangular elements. The left side of the plate is clamped as shown in Fig. 4 (b), and it is assumed that a uniform thermal load of $373 \mathrm{~K}$ is applied. "SPC" in the figure denotes the constraint degree of freedom, i.e. 1 to 3 denote the translational degree of freedom of $x$ to $z$ and 4 to 6 denote the rotational degree of freedom of around $x$ axis to $z$ axis. Figure 5 (a) shows the thermal deformation of a symmetrically laminated plate $\left[0^{\circ} / 90^{\circ} / 0^{\circ}\right]$ for comparison, and Fig. 5 (b) shows the thermal deformation of the unsymmetrically laminated plate. It is confirmed that the symmetrically laminated plate deforms in the in-plane direction, and that the unsymmetrically laminated plate deforms in the out-of-plane direction.

Aiming at reducing the deformation in the out-of-plane direction of the unsymmetrically laminated plate to zero, we set the target value in Eq. (16) to zero, and only the top layer as the design variable. Figure 6 (a) shows the optimal material orientation of the top layer obtained, which is almost the same orientation as the bottom layer as we expected. Figure 6 (b) shows the thermal deformation of the optimized orientation, which is quite similar to the correct solution shown in Fig. 5 (a). Figure 7 shows the iteration history of the objective function normalized to the initial value, and it is confirmed that the objective function monotonically converged to almost zero. The monotonicity of the convergence depends on the amount of variation in one step. To avoid unstable behavior in convergence, we have set a small amount of variation in one step at the expense of the number of convergences. Since this method is a gradient method, if the variation is small, it converges monotonically toward the extreme value. These numerical results show the validity of the proposed method to tailor the thermal deformation to the desired value.

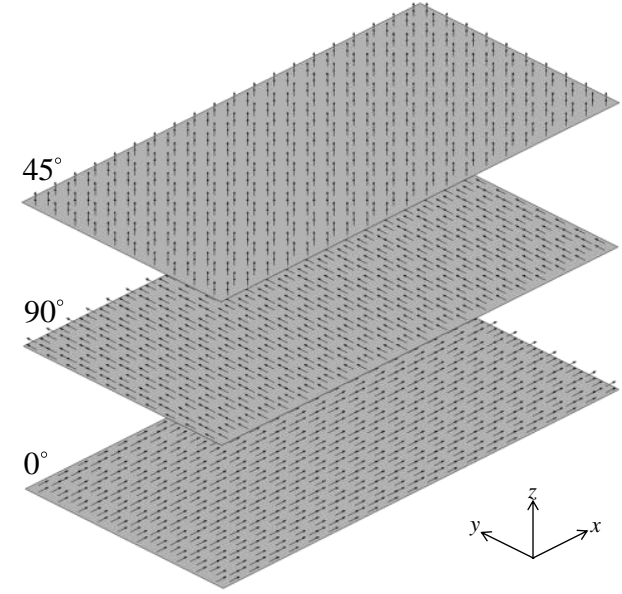

(a) Unsymmetrically laminated plate with 3 layers and material orientation distribution of $E_{1}$

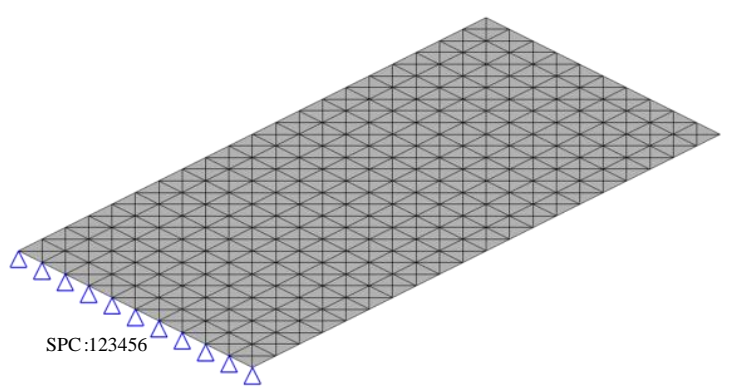

(b) Boundary condition

Fig. 4 Problem definition of three-layer rectangular plate. 


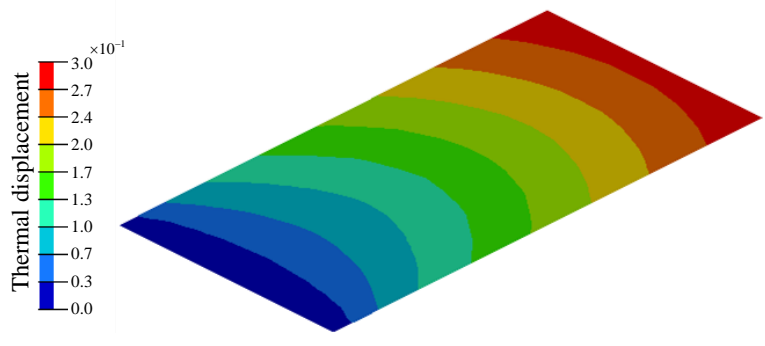

(a) Symmetrical

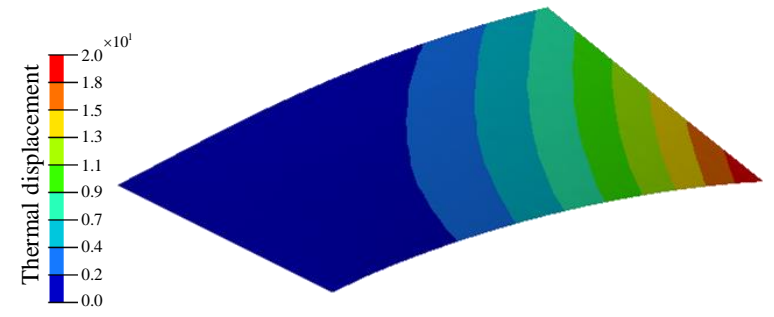

(b) Unsymmetrical

Fig. 5 Comparison of thermal deformations between symmetrically and unsymmetrically laminated plates.

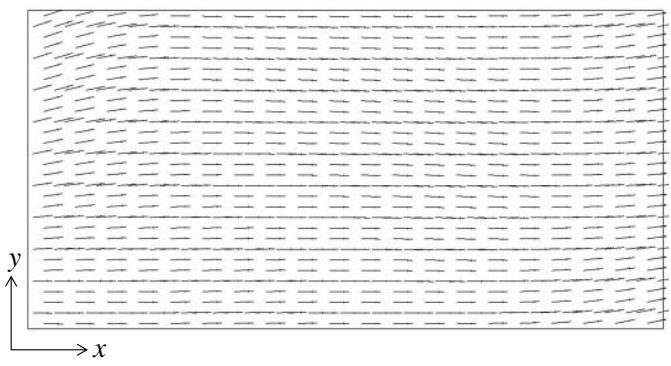

(a) Optimal material-orientation distribution of $E_{1}$ of top layer

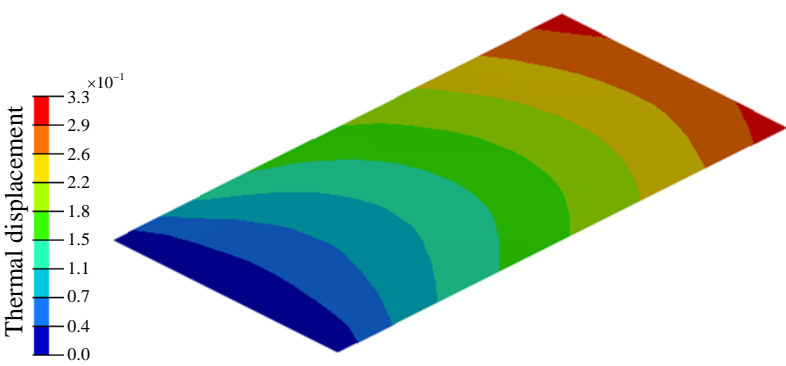

(b) Optimized deformation

Fig. 6 Optimal material orientation of $E_{1}$ on the top layer and its thermal deformation.

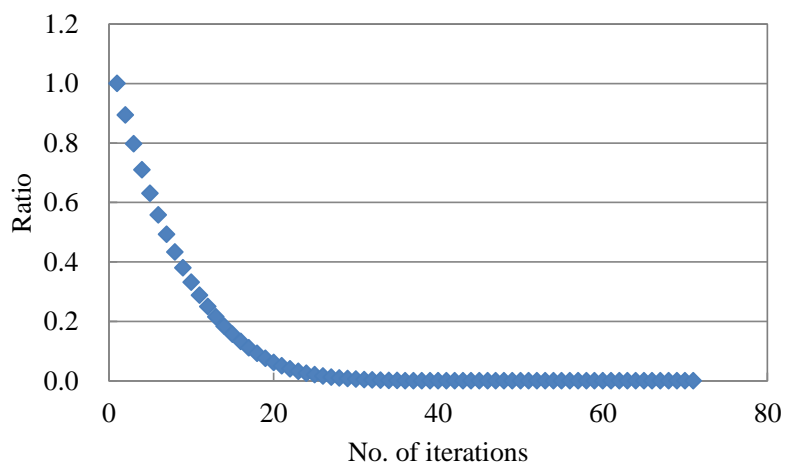

Fig. 7 Iteration history of objective function normalized to the initial value.

\subsection{Three-layer square shell}

\subsubsection{Thermal deformation control of overall displacements}

From a practical point of view, most of the industrial products are exposed in thermal environments, and it is important to maintain the required mechanical performances under thermal stress. Here, consider a 3-layer square shell illustrated in Fig. 8, which is loaded $60 \mathrm{~K}$ temperature difference between the top and bottom surfaces. The four corners are pin-supported. The design domain, which has $200 \mathrm{~mm}$ length in each span and the unit thickness for each layer, has 1600 triangular elements. The initial material orientation of $E_{1}$ is parallel to $x$-axis. The objective of this problem is to reduce the overall thermal displacements to $1 / 10$ of the initial displacement at each 
node. Figure 9 shows the optimal material orientations of $E_{1}$ of each layer, which have different optimal material orientations according to the sensitivity distributions while maintaining their smooth distributions. In particular, the $\mathrm{X}$-type orientation that connects the four constraint points clearly appears in the middle layer. The iteration history of the square displacement error norm normalized to the initial value is shown in Fig. 10(a), and it is confirmed that it is monotonically decreased to $94 \%$. Figures 10(a) and (b) show the comparison of the thermal deformations between the initial and the optimized. Since the four corners are pin-supported, the out-of-plane thermal deformation due to membrane stress and the out-of-plane thermal deformation due to bending stress are superimposed by the influence of the curvature of the shell and the cross-coupling effect. It is confirmed that the thermal deformation is globally reduced by the optimization.

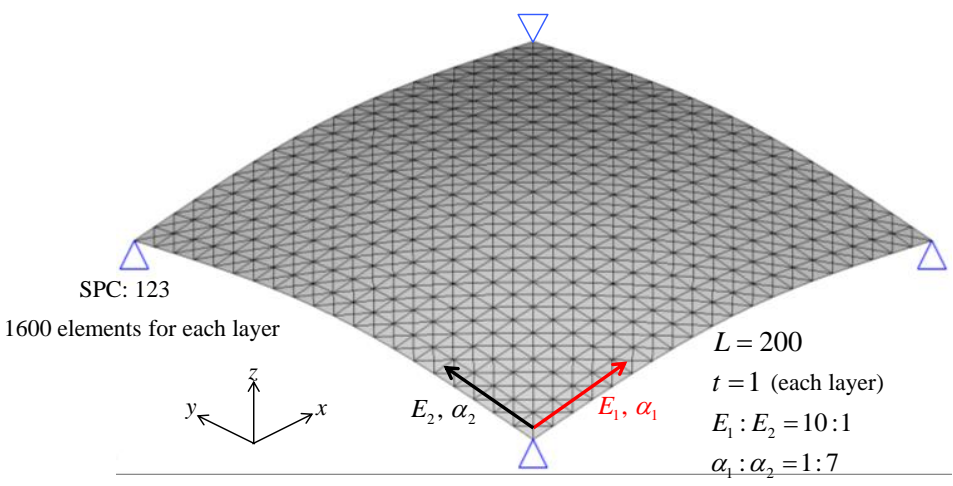

Fig. 8 Boundary conditions of three-layer square shell

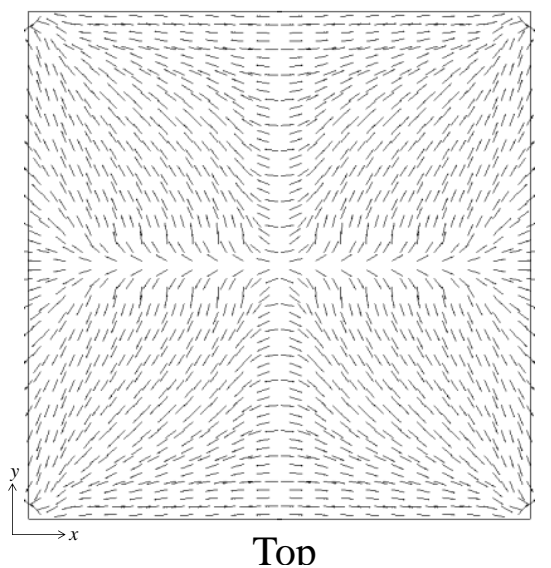

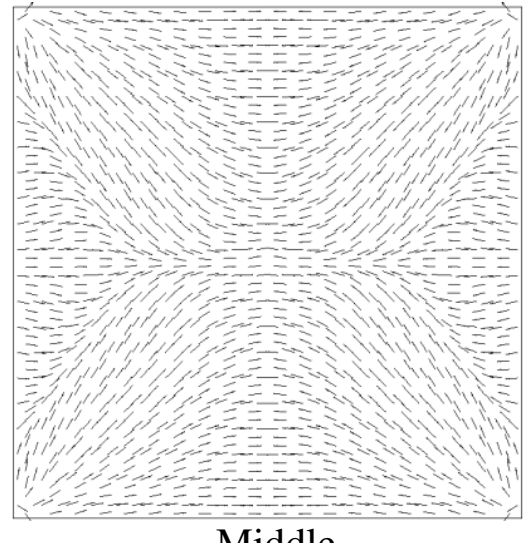

Middle

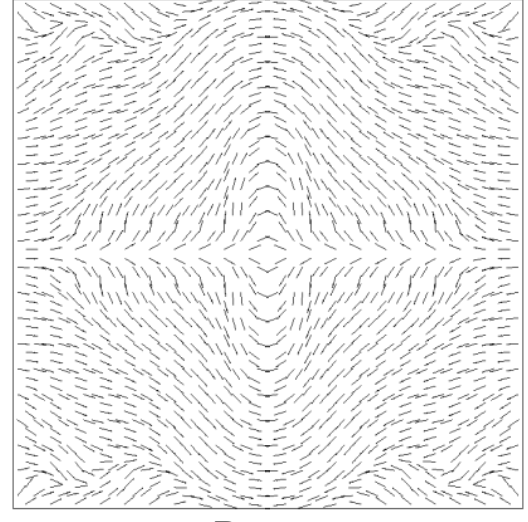

Bottom

Fig. 9 Optimal material orientations of $E_{1}$ of each layer.

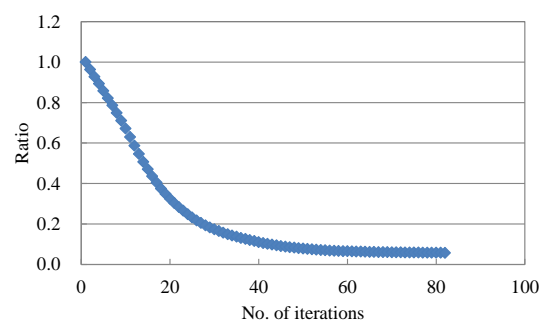

(a) Iteration history of objective function

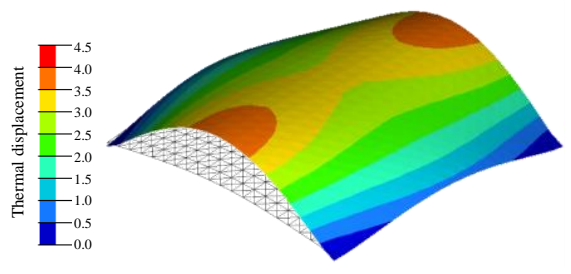

(b) Initial

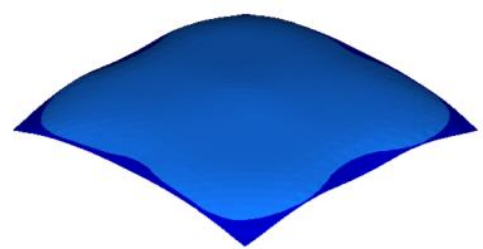

(c) Optimal

Fig. 10 Iteration history and comparison of thermal deformations (indicated by 10 times). 


\subsubsection{Thermal deformation control at specified points}

In the last numerical example, the objective was to reduce the overall thermal displacements to zero. Here, the proposed method is applied to control the displacements at specified points. Figure 11 shows that the problem definition and the boundary conditions, which are same as the last example, but the control points and the target values are different. We set three control points as shown in Fig. 11, and the objective is to reduce the thermal displacement to $1 / 10$ of the initial displacement at three control points. Figure 12 shows the optimal material orientations of $E_{1}$ of each layer obtained, which have different optimal material orientations of $E_{1}$ according to the sensitivity function while maintaining the smooth distributions. Each layer clearly shows an X-type orientation, which is a little bit different from Fig. 9, especially on bottom layer because of the difference of the control points. Figures 13(a), (b) and (c) show the iteration history of the objective function normalized to the initial value and the comparison of the thermal deformations between the initial and the optimized, respectively. It is confirmed that the objective function monotonically converges to zero, and the thermal deformations at the control point become purposely small. These last two numerical examples show validity and utility of the proposed method to tailor the thermal deformation at any point to the desired value.

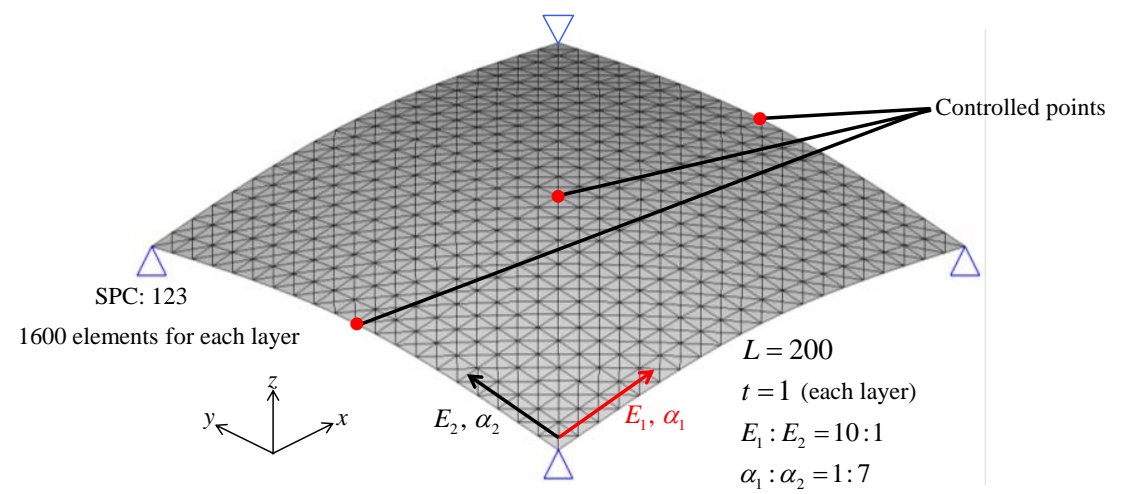

Fig. 11 Problem definition and boundary conditions of three-layer square shell.

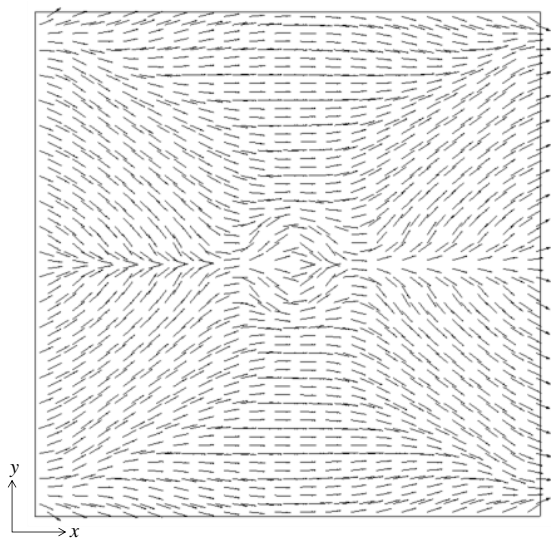

Top

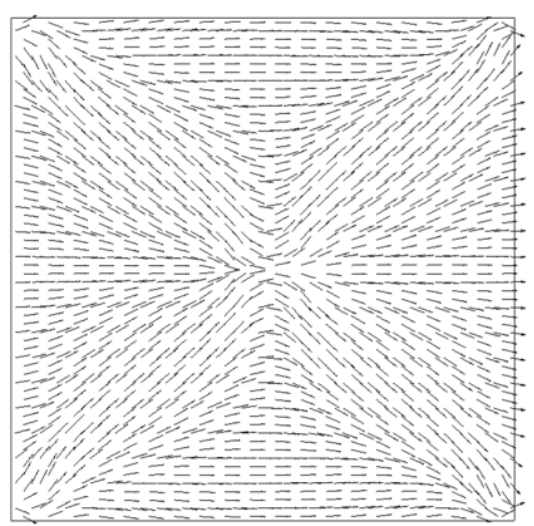

Middle

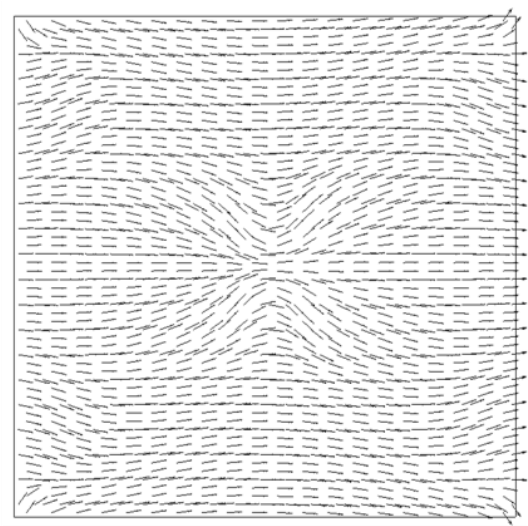

Bottom

Fig. 12 Optimal material orientations of $E_{1}$ of each layer. 


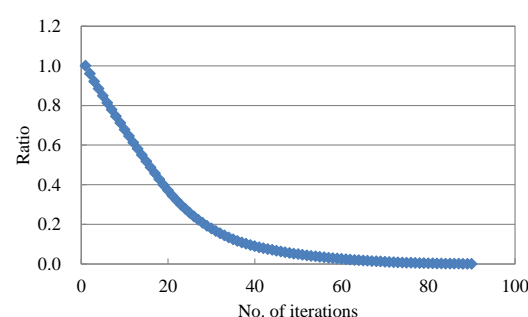

(a) Iteration history of objective

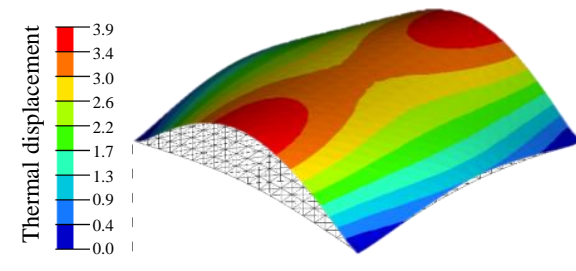

(b) Initial

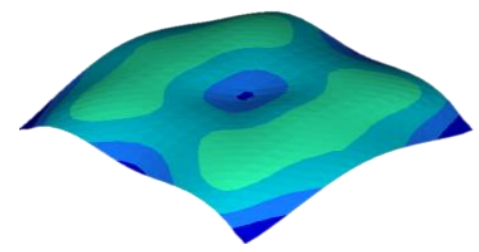

(c) Optimal

Fig. 13 Iteration history and comparison of thermal deformations (indicated by 10 times).

\subsubsection{Static deformation control}

We have treated the thermal deformation control in the last three design examples. Here, we focus on the static deformation control, which can be easily executed by neglecting the terms with respect to the coefficients of thermal expansion $\alpha$ in Eq. (24), to show the ability of the proposed method. The design domain of a squared shell with 3 layers is also used. The problem definition and boundary conditions are shown in Fig. 14, where the four corners are pin-supported and a $100 \mathrm{~N}$ downward concentrated force is applied at its center. The objective of this problem is to control the static deformations in the control region, which is enclosed within the dotted square in red to the target value $-2.4 \times 10^{-2} \mathrm{~mm}$. Figure 15 shows the optimal material orientation of $E_{1}$ on each layer. Different optimal material orientations are obtained according to the sensitivity functions while maintaining the smooth distribution. Figures 16(a), (b) and (c) show the iteration history of objective function normalized to the initial value and the comparison of the static deformations between the initial and the optimized, respectively. It is confirmed that the objective function monotonically converges to zero, and the material orientation around the control region and along the load path have more material variation. It is confirmed that the deformation of the controlled region is successfully tailored to the target value. These results show that the static deformation can be successfully tailored to the target value with the proposed method.

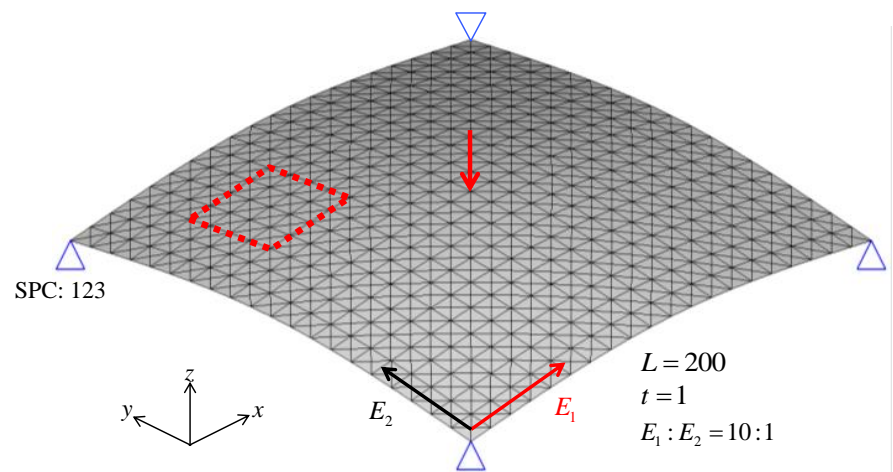

Fig. 14 Problem definition and boundary conditions of three-layer square shell. 


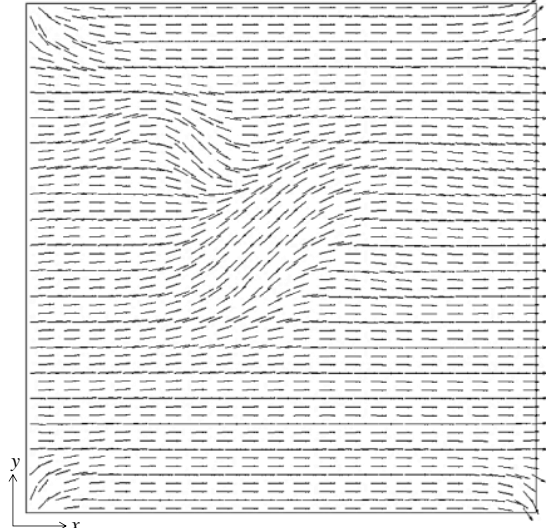

Top

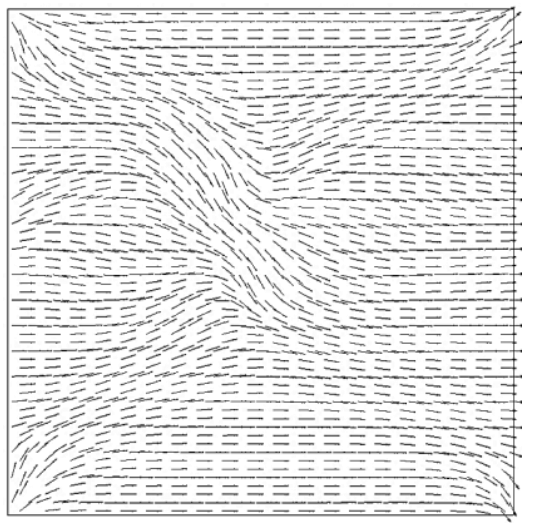

Middle

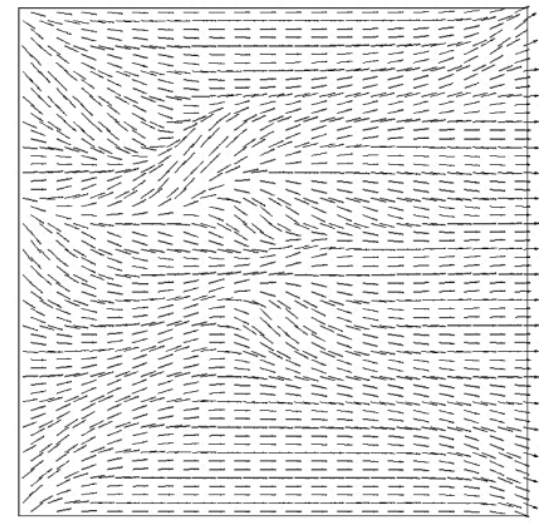

Bottom

Fig. 15 Optimal material orientations of $E_{1}$ of each layer.

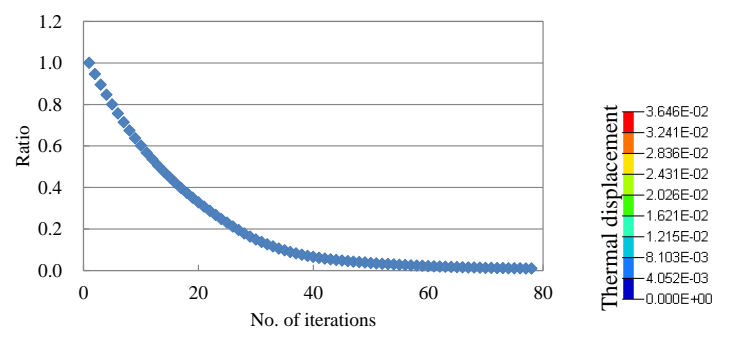

(a) Iteration history of objective function

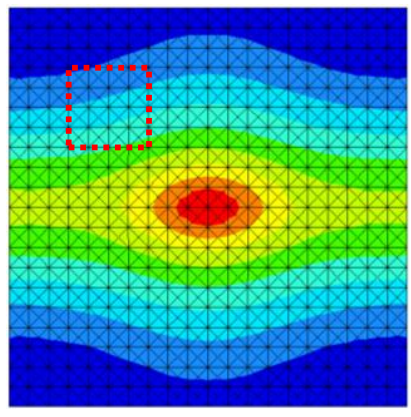

(b) Initial

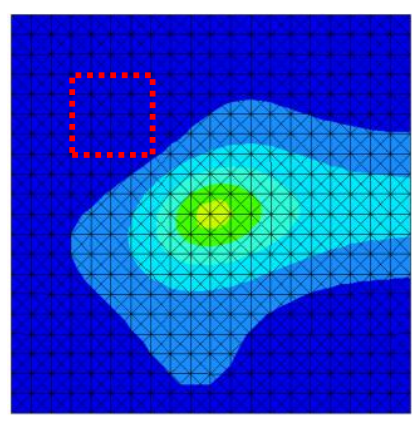

(c) Optimal

Fig. 16 Iteration history and comparison of static deformations (indicated by 10 times).

\subsection{Thermal deformation control of car hood-shaped model}

We apply the proposed method to a car hood-shaped model as a practical example. Figure 17(a) shows a simplified model of car hood, which consists of three layers with orthotropic material. The design domain (approximately 500x300mm) has 1554 triangular elements, and each layer has the unit thickness. The temperature difference between engine-room and outside causes curvature deformation of a car hood, and also results in the reduction of the structural performances such as stiffness, strength, or vibration. As shown in Fig. 17 (a), three points are pin-supported, and $60 \mathrm{~K}$ temperature difference is applied between the top and the bottom surface. The initial material orientation of $E_{1}$ is parallel to $y$-axis. The target deformation is uniformly set to $3.0 \mathrm{~mm}$ in $\mathrm{z}$-axis direction over the controlled area, which is enclosed within the solid line in black as shown in the initial thermal displacements, Fig. 17 (b). Figure 18 shows the optimal material orientation of $E_{1}$ on each layer. Each layer has a complicated material orientation while maintaining the smooth distribution. In particular, the orientation change in the vicinity of the fixed points and the central line with a large curvature is large. Figure 19(a) shows the iteration history of the objective function, and Figs. 19(b) and (c) show the comparison of the initial and the optimized thermal displacements. It is confirmed that the objective function monotonically converges to almost zero, and the thermal deformation in the controlled area becomes uniformly $3.0 \mathrm{~mm}$ as we expected. 


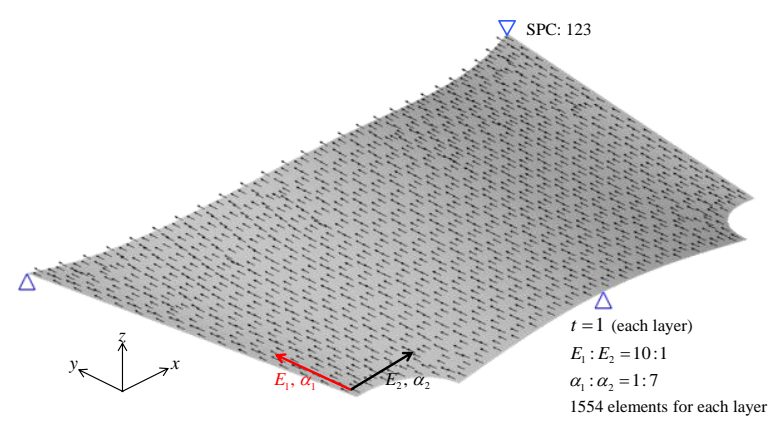

(a) Boundary conditions and initial material orientation of $E_{1}$

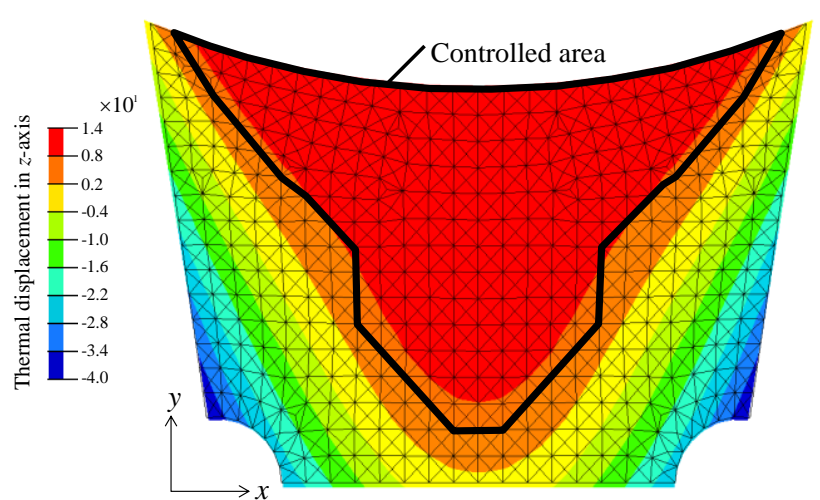

(b) Initial thermal displacement distribution in $\mathrm{z}$-axis and controlled area

Fig. 17 Problem definition of a car hood-shaped model with three layers.

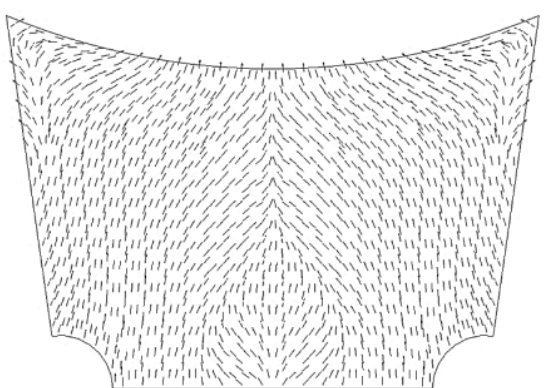

Top

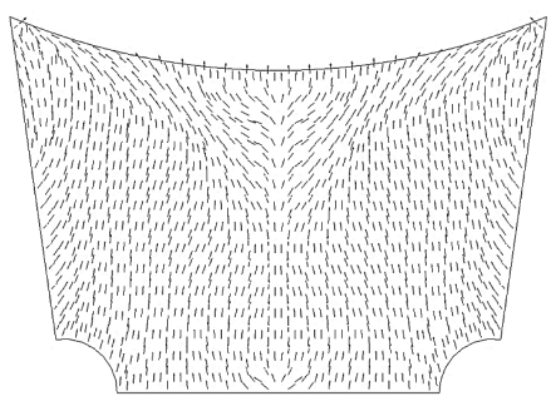

Middle

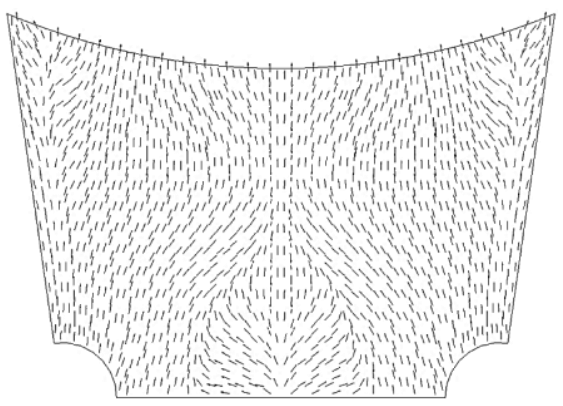

Bottom

Fig. 18 Optimal material orientations of $E_{1}$ of each layer.

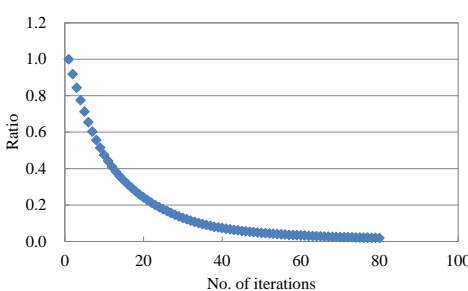

(a) Iteration history of objective function

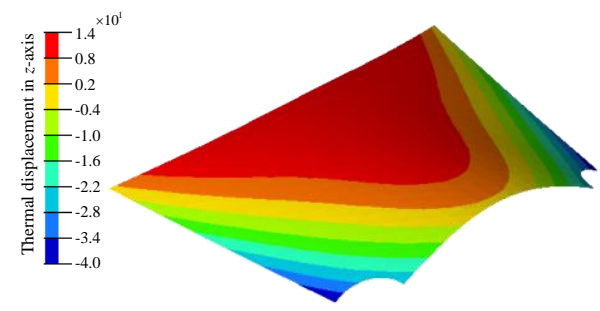

(b) Initial

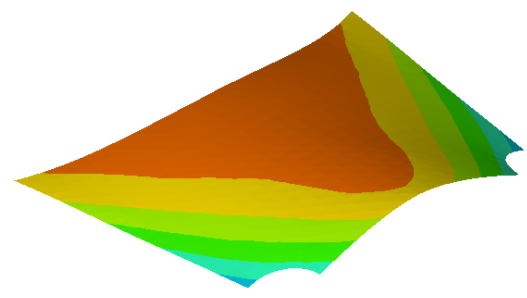

(c) Optimal

Fig. 19 Iteration history and comparison of thermal displacement distributions in z-axis.

\section{Conclusions}

In this paper, we presented a parameter-free optimization method based on the variational method for tailoring thermal or static deformation of laminated composite shell structures. It was assumed that the fiber in a structure consisting of orthotropic materials could be arbitrarily oriented layer by layer, and the each optimum free-orientation 
was determined. The square displacements error norm was used as the objective function. The optimization problem was formulated and the sensitivity function for material orientation variation was theoretically derived using the Lagrange multiplier method and the adjoint variable method. The optimal material orientation was determined with the $\mathrm{H}^{1}$ gradient method with Poisson's equation, where the fictitious heat forces were applied to vary the material orientations. The validity and practical utility of the proposed optimization method were verified through several numerical design examples.

With the proposed approach, the arbitrary and smooth optimal distribution of the material orientation of a laminated anisotropic shell structure could be conventionally created for tailoring the thermal deformation. The proposed method is especially effective for large-scale material-orientation design problems with complicated-shape, and with multi-layer including metal-FRP composites. This method will become an effective tool for designing a high-performance laminated composite shell structure.

Regarding the realization of the obtained fiber orientation, since it will be orientation in free curves, it is difficult to create a continuous tool path that is covered with fiber tape so that all areas have the same thickness. When using the current AFP technology, we believe that the largest possible area will be covered with a continuous tool path of fiber tape and the resulting gap will be covered with an additional tool path. Although the obtained characteristics are expected to be lower than the calculated values, the effect is large compared to the linear orientation. At present, we do not consider the concrete tape width and tow width in actual manufacturing, but we consider this as a future issue.

\section{Appendix A}

Here, we show the stress-strain relations for orthotropic Mindlin-Reissner plates employed in this work. For an orthotropic lamina, the stress-strain relation in the principal material direction (shown in Fig.A1) is given by the following matrix equation with six independent components (Gürdal et al. (1999)).

$$
\left\{\begin{array}{l}
\sigma_{11} \\
\sigma_{22} \\
\tau_{23} \\
\tau_{31} \\
\tau_{12}
\end{array}\right\}=\left[\begin{array}{ccccc}
Q_{11} & Q_{12} & 0 & 0 & 0 \\
Q_{12} & Q_{22} & 0 & 0 & 0 \\
0 & 0 & Q_{44} & 0 & 0 \\
0 & 0 & 0 & Q_{55} & 0 \\
0 & 0 & 0 & 0 & Q_{66}
\end{array}\right]\left\{\begin{array}{l}
\varepsilon_{11} \\
\varepsilon_{22} \\
\gamma_{23} \\
\gamma_{31} \\
\gamma_{12}
\end{array}\right\} .
$$

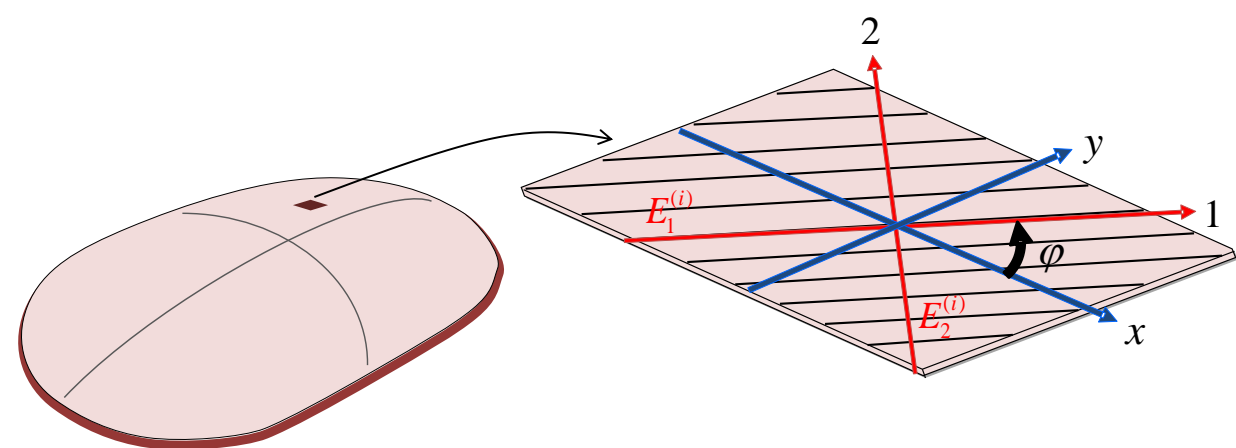

Fig. A1 Definition of material coordinates for orthotropic material.

Note that notation $i$, which indicates $i$-th ply of lamina in previous section, is not shown in this section for simplicity. It is noted that the shear strain is expressed as the engineering shear strain. Each component of the $Q_{i j}$ is a function of the orthotropic material constants, $E_{1}, E_{2}, v_{12}, v_{21}, G_{12}, G_{13}$, and $G_{23}$, and defined as

$$
Q_{11}=\frac{E_{1}}{1-v_{12} v_{21}}, Q_{22}=\frac{E_{2}}{1-v_{12} v_{21}}, Q_{12}=\frac{v_{21} E_{1}}{1-v_{12} v_{21}}=\frac{v_{12} E_{2}}{1-v_{12} v_{21}}, Q_{44}=G_{23}, Q_{55}=G_{13}, Q_{66}=G_{12} \text {. }
$$


Because the orthotropic plates are rotated with respect to a reference coordinate system $x-y$ (shown in Fig. 3), the principle directions of the orthotropic material must be transformed to match the reference axes. The transformed stress-strain relation is given by

$$
\left\{\begin{array}{l}
\sigma_{x x} \\
\sigma_{y y} \\
\tau_{y z} \\
\tau_{x z} \\
\tau_{x y}
\end{array}\right\}=\left[\begin{array}{ccccc}
\bar{Q}_{11} & \bar{Q}_{12} & 0 & 0 & \bar{Q}_{16} \\
\bar{Q}_{12} & \bar{Q}_{22} & 0 & 0 & \bar{Q}_{26} \\
0 & 0 & \bar{Q}_{44} & \bar{Q}_{45} & 0 \\
0 & 0 & \bar{Q}_{45} & \bar{Q}_{55} & 0 \\
\bar{Q}_{16} & \bar{Q}_{26} & 0 & 0 & \bar{Q}_{66}
\end{array}\right]\left\{\begin{array}{c}
\varepsilon_{x x} \\
\varepsilon_{y y} \\
\gamma_{y z} \\
\gamma_{x z} \\
\gamma_{x y}
\end{array}\right\},
$$

where the transformed stiffness matrix $\bar{Q}_{i j}$ is given by

$$
\begin{aligned}
& \bar{Q}_{11}=U_{1}+U_{2} \cos 2 \varphi+U_{3} \cos 4 \varphi, \bar{Q}_{22}=U_{1}-U_{2} \cos 2 \varphi+U_{3} \cos 4 \varphi, \bar{Q}_{12}=U_{4}-U_{3} \cos 4 \varphi \\
& \bar{Q}_{16}=\frac{1}{2} U_{2} \sin 2 \varphi+U_{3} \sin 4 \varphi, \bar{Q}_{26}=\frac{1}{2} U_{2} \sin 2 \varphi-U_{3} \sin 4 \varphi, \bar{Q}_{66}=U_{5}-U_{3} \cos 4 \varphi \\
& \bar{Q}_{44}=Q_{44} \cos ^{2} \varphi+Q_{55} \sin ^{2} \varphi, \bar{Q}_{45}=\frac{1}{2} \sin 2 \varphi\left(Q_{55}-Q_{44}\right),
\end{aligned}
$$

and the elastic invariant $U_{i}(i=1,2, \cdots, 5)$, is given by

$$
\begin{aligned}
& U_{1}=\frac{1}{8}\left(3 Q_{11}+3 Q_{22}+2 Q_{12}+4 Q_{66}\right), U_{2}=\frac{1}{2}\left(Q_{11}-Q_{22}\right), U_{3}=\frac{1}{8}\left(Q_{11}+Q_{22}-2 Q_{12}-4 Q_{66}\right) \\
& U_{4}=\frac{1}{8}\left(Q_{11}+Q_{22}+6 Q_{12}-4 Q_{66}\right), U_{5}=\frac{1}{8}\left(Q_{11}+Q_{22}-2 Q_{12}+4 Q_{66}\right) .
\end{aligned}
$$

The stress-strain relation with respect to the bending and membrane stiffness of orthotropic each layer applies a reduced stiffness of Eq. (A4) and the stress in the $i$-th layer can be expressed in terms of the reduced stiffness of that particular layer as

$$
\left\{\begin{array}{l}
\sigma_{x x} \\
\sigma_{y y} \\
\tau_{x y}
\end{array}\right\}=\left[\begin{array}{lll}
\bar{Q}_{11} & \bar{Q}_{12} & \bar{Q}_{16} \\
\bar{Q}_{12} & \bar{Q}_{22} & \bar{Q}_{26} \\
\bar{Q}_{16} & \bar{Q}_{26} & \bar{Q}_{66}
\end{array}\right]\left(\left\{\begin{array}{c}
\varepsilon_{0 x x} \\
\varepsilon_{0 y y} \\
\gamma_{x y}
\end{array}\right\}+x_{3}\left\{\begin{array}{c}
\kappa_{x x} \\
\kappa_{y y} \\
\kappa_{x y}
\end{array}\right\}\right),
$$

where $\varepsilon_{0}^{(i)}$ and $\boldsymbol{\kappa}^{(i)}$ are the in-plane strain tensor and the curvature tensor in the middle surface of the $i$-th ply, respectively. Furthermore, the stress-strain relation with respect to the shear stiffness of each layer applies a reduced stiffness of Eq. (A4), and the stress in the $i$-th ply can be expressed in terms of the reduced stiffness of that particular layer as

$$
\left\{\begin{array}{l}
\tau_{y z} \\
\tau_{x z}
\end{array}\right\}=\left[\begin{array}{ll}
\bar{Q}_{44} & \bar{Q}_{45} \\
\bar{Q}_{45} & \bar{Q}_{55}
\end{array}\right]\left\{\begin{array}{l}
\gamma_{y z} \\
\gamma_{x z}
\end{array}\right\}
$$

The stress resultants, moment resultants, and transverse shear resultants per unit width of the cross section acting at a point in the laminate are obtained by the through-the-thickness integration of the stresses of each layer. Finally, the stiffness matrices $\boldsymbol{E}^{B(i)}, \boldsymbol{E}^{M(i)}, \boldsymbol{E}^{C(i)}$ and $\boldsymbol{E}^{S(i)}$ on the right side of Eq. (24) can be obtained as follows:

$$
\begin{aligned}
& \left\{E_{\alpha \beta \gamma \delta}^{M}\right\}_{\alpha, \beta, \gamma, \delta=1,2}=\int_{-t_{i} / 2}^{t_{i} / 2}\left[\begin{array}{lll}
\bar{Q}_{11} & \bar{Q}_{12} & \bar{Q}_{16} \\
\bar{Q}_{12} & \bar{Q}_{22} & \bar{Q}_{26} \\
\bar{Q}_{16} & \bar{Q}_{26} & \bar{Q}_{66}
\end{array}\right] d x_{3}, \quad\left\{E_{\alpha \beta \gamma \delta}^{C}\right\}_{\alpha, \beta \gamma, \gamma=1,2}=\int_{-t_{i} / 2}^{t_{i} / 2}\left[\begin{array}{ccc}
\bar{Q}_{11} & \bar{Q}_{12} & \bar{Q}_{16} \\
\bar{Q}_{12} & \bar{Q}_{22} & \bar{Q}_{26} \\
\bar{Q}_{16} & \bar{Q}_{26} & \bar{Q}_{66}
\end{array}\right] x_{3} d x_{3}, \\
& \left\{E_{\alpha \beta \gamma \delta}^{B}\right\}_{\alpha, \beta, \gamma, \delta=1,2}=\int_{-t_{i} / 2}^{t_{i} / 2}\left[\begin{array}{lll}
\bar{Q}_{11} & \bar{Q}_{12} & \bar{Q}_{16} \\
\bar{Q}_{12} & \bar{Q}_{22} & \bar{Q}_{26} \\
\bar{Q}_{16} & \bar{Q}_{26} & \bar{Q}_{66}
\end{array}\right] x_{3}^{2} d x_{3},\left\{E_{\alpha \beta}^{S}\right\}_{\alpha, \beta=1,2}=\int_{-t_{i} / 2}^{t_{i} / 2}\left[\begin{array}{cc}
\bar{Q}_{44} & \bar{Q}_{45} \\
\bar{Q}_{45} & \bar{Q}_{55}
\end{array}\right] d x_{3} .
\end{aligned}
$$

Thus, the distributed material orientation sensitivity function $G_{\varphi}^{(i)}(x)$ is obtained by the derivative of Eq. (A8) with respect to material orientation $\varphi^{(i)}$. Each term of the material orientation sensitivity function derived in Section 2.3 can 
be obtained by calculating the first variation of Eq. (A8). Then, the derivatives of stiffness matrix $\bar{Q}_{i j}$ with respect to $\varphi$ are as follows:

$$
\begin{array}{ll}
\bar{Q}_{11, \varphi}=-2 U_{2} \sin 2 \varphi-4 U_{3} \sin 4 \varphi, \bar{Q}_{22, \varphi}=2 U_{2} \sin 2 \varphi-4 U_{3} \sin 4 \varphi, & \bar{Q}_{12, \varphi}=4 U_{3} \sin 4 \varphi, \\
\bar{Q}_{16, \varphi}=U_{2} \cos 2 \varphi+4 U_{3} \cos 4 \varphi, & \bar{Q}_{26, \varphi}=U_{2} \cos 2 \varphi-4 U_{3} \cos 4 \varphi, \quad \bar{Q}_{66, \varphi}=4 U_{3} \sin 4 \varphi, \\
\bar{Q}_{44, \varphi}=-Q_{44} \sin 2 \varphi+Q_{55} \sin 2 \varphi, & \bar{Q}_{45, \varphi}=\cos 2 \varphi\left(Q_{55}-Q_{44}\right) .
\end{array}
$$

\section{Acknowledgments}

This work was supported by a Grant-in Aid for Scientific Research, Grant Number 18K03853 given by the Japan Society for the Promotion of Science.

\section{References}

Albazzan M A, Harik R. Tatting B F, Gürdal Z, Efficient design optimization of nonconventional laminated composites using lamination parameters: A state of the art, Composite Structures Vol. 209 (2019), pp.362-374.

Ananthasuresh, G., Kota, S. and Kikuchi, N., Strategies for systematic synthesis of compliant mems, ASME Winter Annual Meeting, Vol. 55, No.2 (1994), pp.677-686.

Azegami, H. and Wu, Z. C., Domain optimization analysis in linear elastic problems: approach using traction method, Trans. JSME Ser. A, Vol. 60, No.578 (1994), pp.2312-2318.

Azegami, H., Kaizu, S. and Takeuchi, K., Regular solution to topology optimization problems of continua, JSIAM 3 (2011), pp.1-4.

Guanxin, H., Hu, W. and Guangyao, L., An efficient reanalysis assisted optimization for variable-stiffness composite design by using path functions, Compos. Struct., Vol. 153 (2016), pp.409-420.

Gürdal, Z, Haftka, R. T. and Hajela, P. Design and optimization of laminated composite materials (1999), John Wiley \& Sons.

Hoerner, S. V., Homologous deformations of tiltable telescopes, Proc. ASCE, J Struct Div. 93 (1967), pp.461-485.

Honda, S., Igarashi, T. and Narita, Y., Multi-objective optimization of curvilinear fiber shapes for laminated composite plates by using NSGA-II, Composites Part B:Engineering, Vol. 45, No. 1 (2013), pp.1071-1078.

Hyer, M. W. and Lee, H. H., The use of curvilinear fiber format to improve buckling resistance of composite plates with central circular holes, Compos. Struct. Vol. 18, No. 3 (1991), pp.239-261.

Ikeya, K., Shimoda, M. and Shi, J. X., Multi-objective free-form optimization for shape and thickness of shell structures, Compos. Struct., Vol. 135 (2016), pp.262-275.

Jacob, L. P. and Senthil, S. V., Multi-objective optimization of fiber reinforced composite laminates for strength, stiffness and minimal mass, Comput. Struct., Vol. 84 (2006), pp.2065-2080.

Jung, D. and Gea, H., Compliant mechanism design with non-linear materials using topology optimization, Int. J. Mechan. Mater. Des., Vol. 1, No.2 (2004), pp.157-171.

Kim, J. S., Kim, C. G. and Hong, C. S., Optimal design of composite structures with ply drop using genetic algorithm and expert system shell, Compos. Struct., Vol. 46, No. 2 (1999), pp.171-87.

Kiyono, C. Y., Silva, E. C. N. and Reddy, J. N., A novel fiber optimization method based on normal distribution function with continuously varying fiber path, Compos. Struct. Vol. 160, No.15 (2017), pp.503-515.

Kogiso, N., Watson, L. T., Gürdal, Z. and Haftka, R. T., Genetic algorithms with local improvement for composite laminate design, Structural Optimization, Vol. 7 (1994), pp.207-218.

Le, R. R. and Haftka, R. T., Optimization of laminate stacking sequence for buckling load maximization by genetic algorithm, AIAA Journal Vol. 31 (1993), pp.951-956.

Lee, E. and Gea, H. C., A strain based topology optimization method for compliant mechanism design, Structural and Multidisciplinary Optimization, Vol. 49, No. 2 (2014), DOI: 10.1007/s00158-013-0971-0.

Lee, K. H, Kim, K. K. and Park, G. J., Truss optimization considering homologous deformation under multiple loadings, Struct. Optim. Vol. 16 (1998), pp.193-200. 
Lindgaard, E. and Lund, E., A unified approach to nonlinear buckling optimization of composite structures, Comput. Struct. Vo. 89 (2011), pp.357-370.

Liu, Y. and Shimoda, M., A non-parametric solution to shape identification problem of free-form shells for desired deformation mode, Compos. Struct. Vol. 144 (2014), pp.1-11.

Nishiwaki, S., Frecker, M. I., Min, S. and Kikuchi, N., Topology optimization of compliant mechanisms using the homogenization method, Int. J. Numer. Methods Eng. Vol.42 (1998), pp.535-559.

Muramatsu, Y. and Shimoda, M., Optimization approach for designing free-orientation of orthotropic materials of a shell structure, Transactions of the JSME, Vol. 83, No. 851 (2017) (in Japanese)..

Muramatsu, Y. and Shimoda, M., Distributed-parametric optimization approach for free-orientation of laminated shell structures with anisotropic materials, Struct. Multidisc. Optim., Vol. 59, No. 6 (2019), pp. 1915-1934.

Shimoda, M., Azegami and Sakurai, T., Traction method approach to optimal shape design problems, SAE Trans, J. Passenger Cars, Vol. 106 (1998), pp.2355-2365.

Shimoda, M. and Liu, Y., A non-parametric free-form optimization method for shell structures, Struct. Multidisc. Optim., Vol. 50 (2014), pp.409-423.

Stegmann, J. and Lund, E., Discrete material optimization of general composite shell structures, Int. J. Numer. Methods Eng. Vo. 62, No.14 (2005), pp.2009-2027.

Yamanaka, Y., Todoroki, Y., Ueda, M., Hirano, Y. and Matsuzaki R, Fiber line optimization in single ply for3D printed composites, Open Journal of Composite Materials, Vol. 6, 12-131 (2016). DOI: http://dx.doi.org/10.4236/ojcm.2016.64012.

Yoshikawa, N. and Nakagiri, S., Design change to realize homologous deformation, JSME Int. J. Ser. A, Mechan. Mater. Eng. Vol. 38 (1995), pp.453-457. 